\title{
Ethylene-Responsive Element-Binding Factor 5, ERF5, Is Involved in Chitin-Induced Innate Immunity Response
}

\author{
Geon Hui Son, ${ }^{1,2}$ Jinrong Wan, ${ }^{1}$ Hye Jin Kim, ${ }^{2}$ Xuan Canh Nguyen, ${ }^{2}$ Woo Sik Chung, ${ }^{2}$ Jong Chan Hong, ${ }^{1,2}$ \\ and Gary Stacey ${ }^{1}$
}

\begin{abstract}
${ }^{1}$ Division of Plant Sciences, University of Missouri-Columbia, Columbia 65211, U.S.A.; ${ }^{2}$ Division of Applied Life Sciences (BK21 Program), Plant Molecular Biology and Biotechnology Research Center, Gyeongsang National University, Jinju 660-701, Korea
\end{abstract}

Submitted 13 June 2011. Accepted 10 September 2011.

\begin{abstract}
Our recent work demonstrated that chitin treatment modulated the expression of 118 transcription factor (TF) genes in Arabidopsis. To investigate the potential roles of these TF in chitin signaling and plant defense, we initiated an interaction study among these TF proteins, as well as two chitin-activated mitogen-activated protein kinases (MPK3 and MPK6), using a yeast two-hybrid system. This study revealed interactions among the following proteins: three ethylene-responsive element-binding factors (ERF), five WRKY transcription factors, one scarecrow-like (SCL), and the two MPK, in addition to many other interactions, reflecting a complex TF interaction network. Most of these interactions were subsequently validated by other methods, such as pull-down and in planta bimolecular fluorescence complementation assays. The key node ERF5 was shown to interact with multiple proteins in the network, such as ERF6, ERF8, and SCL13, as well as MPK3 and MPK6. Interestingly, ERF5 appeared to negatively regulate chitin signaling and plant defense against the fungal pathogen Alternaria brassicicola and positively regulate salicylic acid signaling and plant defense against the bacterial pathogen Pseudomonas syringae pv. tomato DC3000. Therefore, ERF5 may play an important role in plant innate immunity, likely through coordinating chitin and other defense pathways in plants in response to different pathogens.
\end{abstract}

Chitin, a $\beta-1,4$ linked polymer of $\mathrm{N}$-acetylglucosamine, is a structural component of fungal cell walls. Chitin fragments (chitooligosaccharides or chitooligomers) released from fungal cell walls can activate various defense responses in plants, such as the induction of reactive oxygen species (ROS), defense genes, and the synthesis of phytoalexins (Shibuya and Minami 2001). Recent studies demonstrated that plants can detect and recognize chitin as a pathogen- or microbe-associated molecular pattern (P/MAMP) via a pattern recognition receptor, LysM RLK1 (also called CERK1), to activate plant defense against an invading fungal pathogen (Iizasa et al. 2009; Miya et al. 2007; Wan et al. 2008b). Based on these findings, a simple linear pathway was tentatively proposed for chitin signaling, starting from the chitin perception by the receptor LysM RLK1 to the activation of a potential mitogen-activated protein

Corresponding author: G. Stacey; E-mail; staceyg@missouri.edu

* The $\boldsymbol{e}$-Xtra logo stands for "electronic extra" and indicates that seven supplementary figures and six supplementary tables are published online and also that Figures 1 and 10 appear in color online. kinase (MPK) cascade and WRKY transcription factors (TF), leading eventually to the induction of chitin-responsive genes $(\mathrm{CRG})$ and defense against fungal pathogens (Wan et al. 2008a).

It is apparent that this linear pathway is oversimplified and many other components remain to be identified. Indeed, increasing evidence suggests that plant signal transduction pathways actually function as complex, interactive networks, with each component in a pathway interacting with multiple proteins (Bader et al. 2008; Grove and Walhout 2008; Zhu et al. 2008). Therefore, a systems approach is needed to better understand the signaling network activated by a particular signal, such as chitin.

TF are well known for their critical role in controlling gene expression and, therefore, are important components in signaling networks (Ciftci-Yilmaz and Mittler 2008; Grove and Walhout 2008; Guzman-Vargas and Santillan 2008). Their functionality in controlling target gene expression is regulated by interactions with other TF or proteins (Grove and Walhout 2008). Various TF interaction networks have been identified for different processes. For instance, members of the WRKY TF family form a transcriptional network to regulate gene expression associated with plant defense (Eulgem 2006; Eulgem and Somssich 2007; Xu et al. 2006). Likewise, transcription factors ABSCISSIC ACID INSENSITIVE3 (ABI3), FUSCA3 (FUS3), and LEAFY COTYLEDON1 and LEAFY COTYLEDON2 (LEC1 and LEC2) form a network to control seed maturation (To et al. 2006), while NPR1 and five WRKY TF form a regulatory network to regulate systemic acquired resistance (SAR) (Wang et al. 2006).

In the case of chitin signaling in plants, an extensive reprogramming of gene expression occurs in response to chitin elicitation (Kaku et al. 2006; Miya et al. 2007; Ramonell et al. 2005; Wan et al. 2008b). This reprogramming of gene expression is likely under the control of multiple TF. Previously, we showed that a number of WRKY TF genes were rapidly and strongly induced by chitin and some appeared to be the potential targets of the upstream MPK3 and MPK6 (Wan et al. 2004). These WRKY TF are potentially involved in controlling the expression of downstream genes, because many CRG contain a W-box in their promoters (Wan et al. 2010) which is specifically recognized and bound by WRKY TF. In order to identify the genome-wide TF potentially involved in regulating chitin signaling, we examined the effect of chitin elicitation on the expression of all the potential TF genes present in the Arabidopsis genome using a quantitative reverse-transcription polymerase chain reaction (qRT-PCR) primer library (corresponding to more than 2,000 TF genes) (Libault et al. 2007). 
The study identified a total of $118 \mathrm{TF}$ genes significantly regulated by chitin treatment, which encode 27 AP2/EREBP TF, 14 WRKY TF, 14 C2H2 zinc-finger TF, 11 MYB TF, and other TF family members (Libault et al. 2007). These data suggest that multiple TF are potentially involved in reprogramming gene expression in response to chitin to mediate plant defense. Although not directly tested, it is likely that some of these TF are also involved in regulating plant defense responses to other P/MAMPs, such as flagellin and elongation factor-Tu, because there is a significant overlap in the gene expression profiles induced by these different elicitors (Wan et al. 2008a,b ; Zipfel et al. 2006).

In order to reveal the potential roles of these chitin-responsive TF genes in chitin signaling and plant defense, we conducted a large-scale, pairwise yeast two-hybrid (Y2H) interaction screen with the proteins encoded by these 118 TF genes, as well as two MPK (MPK3 and MPK6), previously shown to be activated by chitin (Wan et al. 2004). We found that different TF can interact with other TF, themselves, or with the MPK. Here, we report an interaction network involving three ethyleneresponsive element-binding factor (ERF) TF, five WRKY TF, one scarecrow-like (SCL) TF, and two MPK. Interestingly, the key node ERF5, which interacted with ERF6, ERF8, and SCL13, as well as MPK3 and MPK6, appeared to negatively regulate chitin signaling and plant defense against the fungal pathogen Alternaria brassicicola, and positively regulate salicylic acid (SA) and plant defense against the bacterial pathogen Pseudomonas syringae pv. tomato DC3000. Additionally, ERF5 also appeared to positively regulate ethylene (ETH) signaling. Therefore, ERF5 may play an important role in plant defense, possibly through differentially regulating chitin and other defense pathways in plants in response to different pathogens.

\section{RESULTS}

\section{Y2H screen for interactions among TF and MPK.}

As a prelude to examining protein interactions, we succeeded in cloning most of the predicted full-length cDNAs for

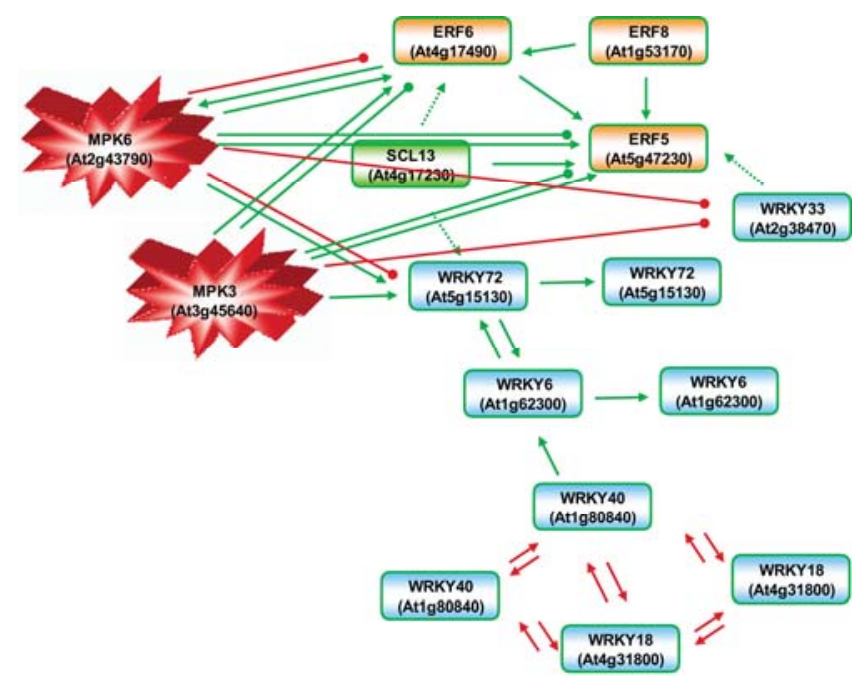

Fig. 1. Interaction network composed of two mitogen-activated protein kinases (MPK) and nine transcription factors (TF). Lines with an arrowhead indicate interactions initially revealed by yeast two-hybrid $(\mathrm{Y} 2 \mathrm{H})$, and the lines with a ball indicate the phosphorylation of TF by MPK. Solid lines indicate that interactions or phosphorylation events were validated with more than one method or by more than one laboratory, and dashed lines indicate those events that were not validated. The direction of the arrow between two proteins indicates that the protein fused to the DNA binding domain interacts with the other protein fused to the activation domain. each of the 118 chitin-responsive TF genes (Libault et al. 2007; Wan et al. 2004), as well as MPK3 and MPK6 (Wan et al. 2004), into the $\mathrm{Y} 2 \mathrm{H}$ bait and prey vectors, respectively (discussed below). Those TF constructs showing autoactivation (as the bait) were excluded from further screening (Supplementary Table 1), leaving a total of $68 \mathrm{TF}$ genes (without autoactivation) in the bait vector (Supplementary Table 2) and two MPK genes for further screening. Eventually, the Y2H screen identified 69 interaction pairs (Supplementary Table 3). Among these interactions, we noticed the following interaction pairs due to their proven or implicated role in plant defense: MPK3/ERF5, MPK3/ERF6, MPK3/WRKY72, MPK6/ERF5, MPK6/ERF6, MPK6/WRKY72, ERF5/ERF6, ERF5/ERF8, ERF6/ERF8, ERF5/WRKY33, ERF5/SCL13, ERF6/SCL13, SCL13/WRKY72, WRKY72/WRKY6, WRKY6/WRKY40, WRKY40/WRKY18, WRKY18/WRKY18, WRKY6/WRKY6, WRKY40/WRKY40, and WRKY72/WRKY72 (Supplementary Fig. 1). Based on these interactions, an interaction network was constructed and shown graphically in Figure 1. Because the majority of the proteins (i.e., WRKY6, WRKY18, WRKY33, WRKY40, and WRKY72, as well as MPK3 and MPK6), were previously shown to be involved in plant defense (Agarwal et al. 2006; Asai et al. 2002; Bhattarai et al. 2010; Broekaert et al. 2006; Eulgem and Somssich 2007; Lippok et al. 2007; Nakano et al. 2006; Pandey and Somssich 2009; Pre et al. 2008; Qiu et al. 2008; Zheng et al. 2006), we reasoned that this whole network could play an important role in chitin signaling and plant defense. Therefore, this network became the focus of efforts to validate the $\mathrm{Y} 2 \mathrm{H}$-predicted interactions and to examine their role in P/MAMP signaling in this study.

\section{Validation of the TF/TF interactions identified by the Y2H screen.}

Because $\mathrm{Y} 2 \mathrm{H}$ screens tend to produce false-positive interactions, the interaction data presented in Figure 1 need to be validated by other methods. Therefore, we made His- and GSTtagged recombinant proteins in Escherichia coli for ERF5, ERF8, WRKY6, WRKY40, and SCL13 and then used these proteins in pull-down experiments. These experiments showed

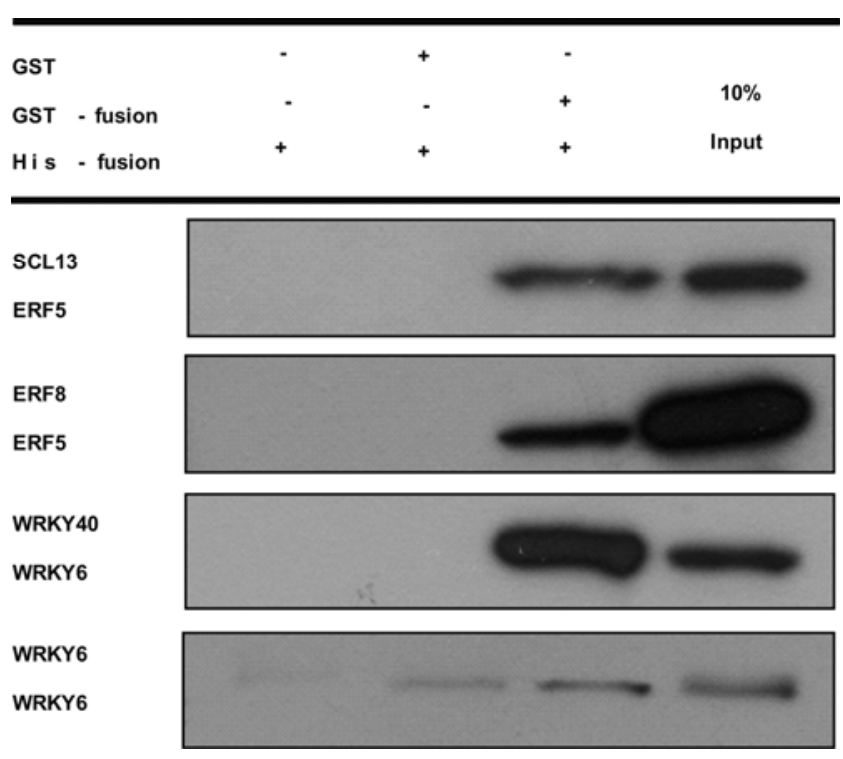

Fig. 2. In vitro pull-down assay to validate yeast two-hybrid data. Selected proteins were expressed as either GST fusion proteins or as His-tagged proteins. To test the in vitro interaction between two proteins, a purified GST-tagged protein (bound to glutathione sepharose beads) was incubated with a His-tagged protein. Co-precipitated proteins were separated on a sodium dodecyl sulfate-polyacrylamide gel and detected by Western blotting with an anti-His antibody. 
that the following His-tagged TF were co-precipitated with the corresponding GST-tagged TF by glutathione sepharose beads, which can specifically bind the GST tag: His-ERF5/GSTSCL13, His-ERF5/GST-ERF8, His-WRKY6/GST-WRKY40, and His-WRKY6/GST-WRKY6. Control experiments showed that none of the His-tagged proteins were co-precipitated with GST in the presence of glutathione sepharose beads (Fig. 2). Therefore, this experiment validated the following interactions: ERF5/SCL13, ERF5/ERF8, WRKY6/WRKY40, and WRKY6/WRKY6.

However, in vitro evidence for protein-protein interaction may not reflect true in planta interactions. Therefore, to examine in planta interactions, we chose the bimolecular fluorescence complementation (BiFC) technique, which enables direct visualization of protein-protein interactions in living cells. The BiFC assay is based on the fact that fluorescence is generated from two nonfluorescent fragments derived from a fluorescent protein (such as the yellow fluorescent protein [YFP]) when they are brought into close proximity to each other due to protein-protein interactions (Kerppola 2008, 2009). We employed a split-YFP-based BiFC system, kindly provided by S. Gelvin at Purdue University (Citovsky et al. 2006). We fused each of the potentially interacting partners to the N-terminal (nYFP) or C-terminal (cYFP) fragment of YFP and then co-infiltrated the Agrobacterium tumefaciens strains containing each of the corresponding fusion constructs into Nicotiana benthamiana leaves to test their in planta interactions. In planta interactions were seen with the following pairs, as reflected by the fluorescent signal in the infiltrated tobacco cells (Fig. 3): ERF6/ERF5, ERF6/ERF8, SCL13/ERF5, WRKY72/WRKY72，WRKY72/

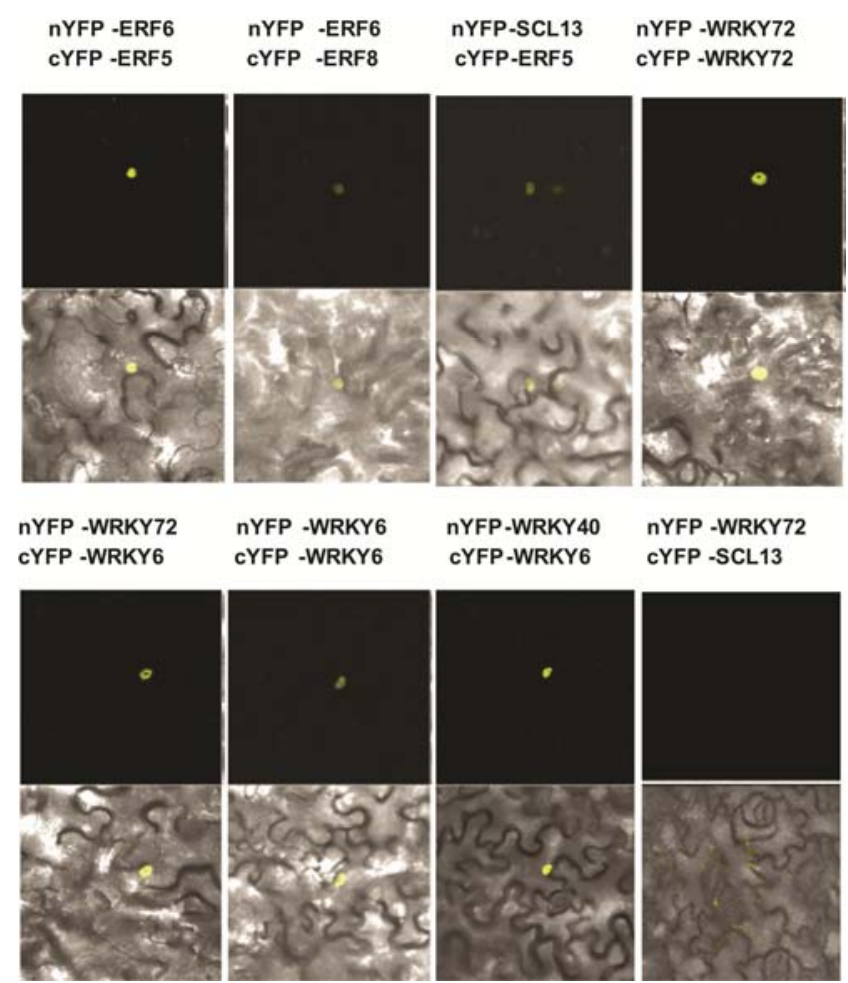

Fig. 3. Application of bimolecular florescence complementation (BiFC) to validate the interactions between transcription factors (TF). The Agrobacterium strain harboring different BiFC constructs as indicated above each image was co-infiltrated into Nicotiana benthamiana leaves to examine TF-TF interactions. In each image, top panel = epifluorescence image and bottom panel $=$ merged with the brightfield image. The yellow fluorescent signal indicates the interaction. The experiment was repeated multiple times with similar results. Additionally, similar results were observed from multiple infiltration sites from the same construct pairs. nYFP and cYFP = $\mathrm{N}$-terminal and $\mathrm{C}$-terminal yellow fluorescent protein, respectively.
WRKY6, WRKY6/WRKY6, and WRKY40/WRKY6. However, we did not see the interaction between WRKY72 and SCL13 in the BiFC experiment. As would be expected for TF proteins, in all cases, the proteins appeared to interact in the nuclei of epidermal cells (Fig. 3), consistent with their subcellular localization shown by the green fluorescent protein-fusion proteins (Supplementary Fig. 2). VirD2/IMP $\alpha$, included as a positive control, was also shown to interact in the nucleus, as revealed previously (Bhattacharjee et al. 2008). Meanwhile, no fluorescent signal was observed in tobacco leaf cells infiltrated with either a single nYFP-TF or cYFP-TF fusion construct or a cYFP-TF or nYFP-TF fusion construct plus nYFPVirD2 or cYFP-IMP $\alpha$ (Supplementary Fig. 3). The experiment was repeated at least three times with similar results. Additionally, similar results were also observed from two to three leaf locations infiltrated with the same construct pair. Therefore, the above BiFC experiments not only validated the following interactions but also supported that these interactions occur in plant cells: ERF6/ERF5, ERF6/ERF8. SCL13/ERF5, WRKY72/WRKY72, WRKY72/WRKY6, WRKY6/WRKY6, and WRKY40/WRKY6. Noteworthily, three interactions (SCL13/ERF5, WRKY40/WRKY6, and WRKY6/WRKY6) have been validated by both in vitro pull-down and in planta BiFC experiments.

To test whether the chitin treatment affects the above interactions, we pretreated the tobacco leaves for 30 min with chitin and then examined the protein-protein interactions using BiFC for the following pairs: ERF6/ERF5, SCL13/ERF5, WRKY6/ WRKY6, WRKY40/WRKY6, and WRKY72/WRKY6. We found that the chitin treatment did not affect their interactions (Supplementary Fig. 4) and the results were similar to those obtained from the untreated plants (Fig. 3). However, we can not rule out that chitin elicitation could affect protein interactions if tested in Arabidopsis. Additionally, the infiltration of tobacco leaves with the Agrobacterium strains likely induces a massive defense reaction, which may complicate the interaction between the tested proteins.

\section{Validation of the MPK/TF interactions by in vitro phosphorylation.}

The interactions between MPK and their potential substrates are likely transient and, therefore, hard to visualize with most methods. In order to validate the interactions between the MPK and TF shown in Figure 1, we tested the ability of MPK3 and MPK6 to directly phosphorylate various recombinant TF proteins expressed in E. coli. These experiments showed that both GST-ERF5 and GST-ERF6 were radioactively labeled by ${ }^{32} \mathrm{P}$ in the presence of $\gamma^{32} \mathrm{P}$-ATP and MPK3 or MPK6, which was expressed as a His-tagged recombinant protein in E. coli. GST alone (as a negative control) was not labeled by ${ }^{32} \mathrm{P}$ in the presence of the recombinant MPK3 or MPK6 protein. Meanwhile, myelin basic protein (a commonly used substrate for kinases) was also labeled by ${ }^{32} \mathrm{P}$ in the presence of the recombinant MPK3 or MPK6 protein. Some smaller bands in the GST-ERF6 lanes are likely degradation protein products from GST-ERF6. Therefore, as predicted by the $\mathrm{Y} 2 \mathrm{H}$ experiments, these data support a direct interaction between MPK3 and MPK6 and ERF5 and ERF6 resulting in phosphorylation (Fig. 4). Consistent with this, both ERF5 and ERF6 contain a putative MPK target site (PXXSPXSP) (X represents any amino acid) in their C-termini (Fujimoto et al. 2000; Pearson and Kemp 1991).

Due to the difficulty of expressing the WRKY72 protein in $E$. coli, we could not examine the phosphorylation of WRKY72 by MPK3 and MPK6. However, Popescu and colleagues (2008) recently reported phosphorylation of WRKY72 by MPK6 using a functional protein microarray. Additionally, these researchers also showed that ERF6 was phosphorylated by MPK6 (Popescu 
et al. 2008), consistent with our current findings. The phosphorylation of chitin-responsive TF by MPK3 and MPK6 suggests that MPK/TF interactions may play an important role in relaying the chitin signal to downstream $\mathrm{CRG}$.

In summary, we succeeded in validating most of the interactions illustrated in Figure 1 by using other methods, as color coded in Figure 1. Additionally, the following interactions revealed by other researchers were also included and color coded in the figure and in Supplementary Table 4: WRKY40/ WRKY18, WRKY72/MPK6, WRKY33/MPK3, and WRKY33/ MPK6 (Mao et al. 2011; Popescu et al. 2008; Xu et al. 2006). Our data now suggest that these published interactions are themselves part of a larger interaction network, as depicted in Figure 1.

As mentioned previously, the majority of the proteins (WRKY6, WRKY18, WRKY33, WRKY40, and WRKY72, and MPK3 and MPK6) in the network are involved in plant disease resistance (Agarwal et al. 2006; Asai et al. 2002; Bhattarai et al. 2010; Broekaert et al. 2006; Eulgem and Somssich 2007; Lippok et al. 2007; Nakano et al. 2006; Pandey and Somssich 2009; Pre et al. 2008; Qiu et al. 2008; Zheng et al. 2006). However, there are no published reports implicating the other proteins (ERF5, ERF6, and ERF8, and SCL13) in this role. Because both ERF5 and ERF6 are capable of interacting with multiple proteins in the TF network, we decided to focus on these proteins to test their potential involvement in plant innate immunity. In addition to their occurrence in the TF network, ERF5 and ERF6 were also selected for their potential role in regulating the ETH signaling pathway, which is involved in plant defense (Broekaert et al. 2006; Fujimoto et al. 2000).

\section{Characterization of the ERF5 and ERF6 mutant plants and transgenic plants ectopically expressing $E R F 5$ and an $E R F 5-E A R$ construct.}

The ERF5 gene (At5g47230) is 903 bp long and lacks introns. The coding sequence is predicted to encode a TF of 300 amino acids, with one ERF/APETALA2 (AP2) domain (Fig. 5A). Similarly, the ERF6 gene (At4g17490) is 849 bp long and also lacks introns. It is predicted to encode a TF of 282 amino acids, with one ERF/AP2 domain (Fig. 5F). Both ERF5 and ERF6 belong to the ERF subfamily B-3 of the ERF/AP2 transcription factor family (Nakano et al. 2006) and are very similar to each other, with $58.0 \%$ identity at the amino acid level.

In order to investigate the potential role of ERF5 in chitin signaling and plant defense, we obtained a homozygous T-DNA insertion mutant (erf5-1) (Fig. 5A) and showed that the insertion indeed blocked the ERF5 expression using RT-PCR (Fig. 5B). Additionally, the qRT-PCR analysis of the mutant also showed that the expression of ERF5 was hard to detect in the mutant (Supplementary Fig. 5A). In order to partially mimic the effect caused by the T-DNA insertion in the ERF5 gene, we also generated transgenic plants ectopically expressing an ERF5-EAR fusion construct (ERF5-EAR plants) under the control of the strong, constitutive $35 \mathrm{~S}$ promoter derived from the Cauliflower mosaic virus (CaMV) 35S RNA (Fig. 5C). The EAR motif is a repression domain that can convert a TF protein, which is a positive regulator of gene expression, into a suppressor of gene expression (Hiratsu et al. 2003; Ohta et al. 2001). As shown by RTPCR (Fig. 5D), the expression of ERF5 was indeed elevated in the selected homozygous ERF5-EAR plants (13 and 26). Additionally, the qRT-PCR analysis of these plants also showed a significant increase in the expression of ERF5 compared with wildtype plants. We also created transgenic plants ectopically expressing ERF5 under the control of the CaMV 35S promoter (ERF5-OX plants). The expression of ERF5 was shown to be elevated in the selected homozygous ERF5-OX plants by RTPCR (Fig. 5E) and qRT-PCR.
A homozygous ERF6 insertion mutant (erf6-1) was also obtained (Fig. 5F) and the expression of ERF6 was blocked in this mutant as shown by RT-PCR (Fig. 5G) and qRT-PCR.

ERF5 and ERF6 are very similar at the amino acid level, interact with each other in the network, and are co-expressed in a large number of microarray experiments, with a correlation efficient of 0.83, as analyzed using ATTED II (an Arabidopsis thaliana trans-factor and cis-element prediction database) (Obayashi et al. 2007, 2009). This suggests that these two genes may be functionally redundant. Therefore, we also generated a double mutant homozygous for both genes by crossing the single insertion mutants erf5-1 and erf6-1. Overall, the various transgenic and mutant plants appeared to be morphologically normal. However, plants ectopically expressing ERF5 appeared to flower slightly earlier than wild-type plants, and the mutant plant erf5-1 appeared to flower slightly later than wild-type plants. Additionally, the rosette leaves of the ERF5 OX plants also appeared to be slightly bigger (Supplementary Fig. 6).

The same set of plants consisting of the wild-type, ERF5-OX (37 and 38), erf5-1, erf6-1, erf5-1/erf6-1, and ERF5-EAR (13 and 26) plants was employed in the following experiments to examine the potential involvement of ERF5 in defense signaling and innate immunity. Briefly, using these plants, we examined the following effects of the manipulation of ERF5 on i) the ex-

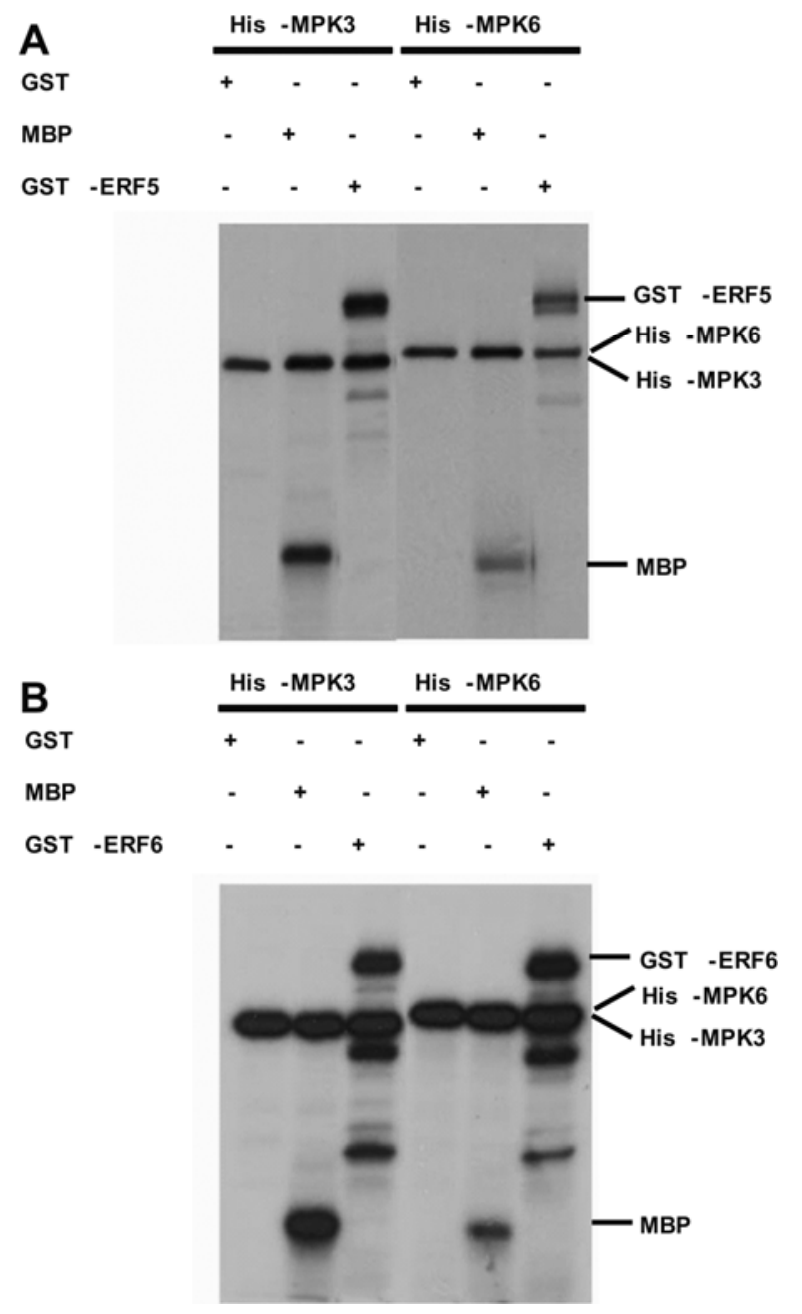

Fig. 4. Phosphorylation of ethylene-responsive element-binding factor (ERF) 5 and EFR6 by mitogen-activated protein kinase (MPK)3 and MPK6. His-tagged MPK3 and MPK6 proteins were purified from Escherichia coli to test their ability to phosphorylate A, GST-ERF5 and B, GSTERF6. GST and myelin basic protein were also included in the experiment to serve as a negative and a positive control, respectively. 
pression of chitin-responsive genes; ii) the infection by a fungal pathogen (Alternaria brassicicola); iii) the infection by a bacterial pathogen ( $P$. syringae pv. tomato DC3000); iv) the expression of SA-, jasmonic acid (JA)/ETH-pathway genes; and v) the triple response.

Differential induction of CRG by chitin in wild-type, ERF5-OX, erf5-1, erf6-1, erf5-1/erf6-1, and $E R F 5-E A R$ plants.

To test the potential role of ERF5 and ERF6 in chitin signaling, we compared the expression of selected CRG (WRKY33 and WRKY53 and MPK3) (Wan et al. 2004, 2008b) in response to the chitin treatment in the wild-type, ERF5-OX (37 and 38), erf5-1, erf6-1, erf5-1/erf6-1, and ERF5-EAR (13 and 26) plants using qRT-PCR. These comparisons showed that the induction of the CRG by chitin in the ERF5-OX (37 and 38) plants was significantly reduced compared with that in the wild type, except that the reduction of WRKY53 induction by chitin in ERF5$O X 38$ was not statistically significant (Fig. 6), suggesting that ERF5 may negatively regulate the induction of these CRG in response to chitin.

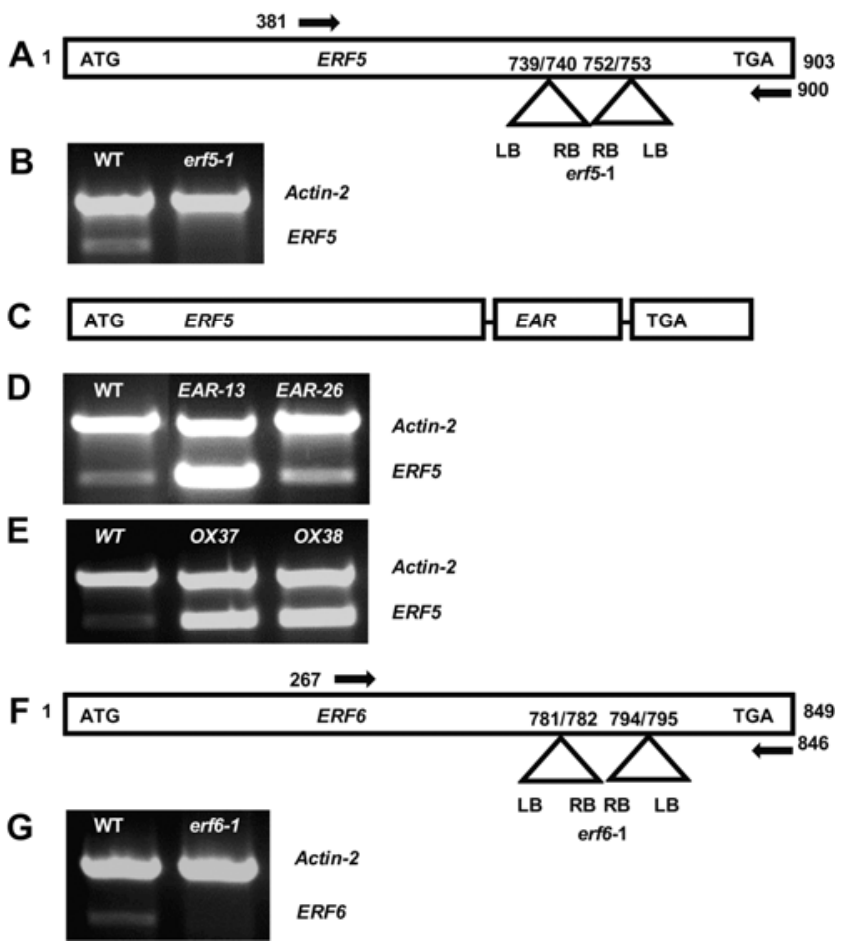

Fig. 5. Analyses of the ethylene-responsive element-binding factor ( ERF)5 and ERF6 insertion mutants, ERF5-EAR, and Cauliflower mosaic virus (CaMV) 35S ERF5 plants. A, Schematic representation of the ERF5 open reading frame (ORF) and T-DNA insertion mutant (erf5-1) (not drawn to scale). Primers for checking the gene expression were represented by the black arrows. Numbers indicate nucleotide positions. B, Reverse-transcription polymerase chain reaction (RT-PCR) analysis of the ERF5 expression in the wild-type (WT) and insertion mutant (erf5-1) plants using the primers indicated in A. C, Schematic representation of the ERF5-EAR fusion construct (not drawn to scale). Primers for checking the gene expression were represented by the black arrows. Numbers indicate nucleotide positions. D, RT-PCR analysis of the ERF5-EAR expression in the selected transgenic plants, using the primers indicated in A. E, RTPCR analysis of the ERF5 expression in the CaMV 35S (ERF5-OX) transgenic plants using the primers indicated in A. F, Schematic representation of the ERF6 ORF and T-DNA insertion mutant (erf6-1) (not drawn to scale). Primers for checking the gene expression were represented by the black arrows. Numbers indicate nucleotide positions. G, RT-PCR analysis of the ERF6 expression in the WT and insertion mutant (erf6-1) plants, using the primers indicated in C. Actin-2 served as an internal control in B, $\mathrm{D}, \mathrm{E}$, and $\mathrm{G}$.
Differential responses to the fungal pathogen

$A$. brassicicola by wild-type, ERF5-OX, erf5-1, erf6-1, erf5-1/erf6-1, and ERF5-EAR plants.

Because chitin is a P/MAMP produced by pathogenic fungi and can trigger plant defense, and because ERF5 and ERF6 are strongly induced by chitin, an obvious question is whether ERF5 or ERF6 plays a role in fungal pathogenicity. To address this question, we tested the virulence of the necrotrophic fungal pathogen A. brassicicola on wild-type, ERF5-OX (37 and 38), erf5-1, erf6-1, erf5-1/erf6-1, and ERF5-EAR (13 and 26) plants. Transgenic plants ectopically expressing ERF5 (ERF5-OX) were much more susceptible to the fungal pathogen than the wild type (Fig. 7), as reflected by more severe symptoms (bigger lesion size and more chlorosis) and the production of more fungal spores per lesion (Fig. 7). The single insertion mutants erf5-1 and erf6- 1 showed a response similar to that of wild-type plants. However, the double insertion mutant erf5-1/erf6-1, as well as the ERF5-EAR plants, showed a slightly enhanced resistance to the fungal pathogen compared with wild-type plants (Fig. 7). These results suggest that ERF5 (and perhaps also ERF6) negatively regulates plant defense against this fungal pathogen. Therefore, ERF5 appears to not only negatively regulate the induction of some CRG by chitin but also negatively regulates plant defense against a fungal pathogen.

\section{Differential responses to the bacterial pathogen $P$. syringae pv. tomato DC3000 by wild-type, ERF5-OX, erf5-1, erf6-1, erf5-1/erf6-1, and ERF5-EAR plants.}

The above fungal pathogen assays suggest that ERF5 is a negative regulator of plant defense against the necrotrophic fungal pathogen A. brassicicola. However, is ERF5 also involved in plant defense against biotrophic pathogens? To an-

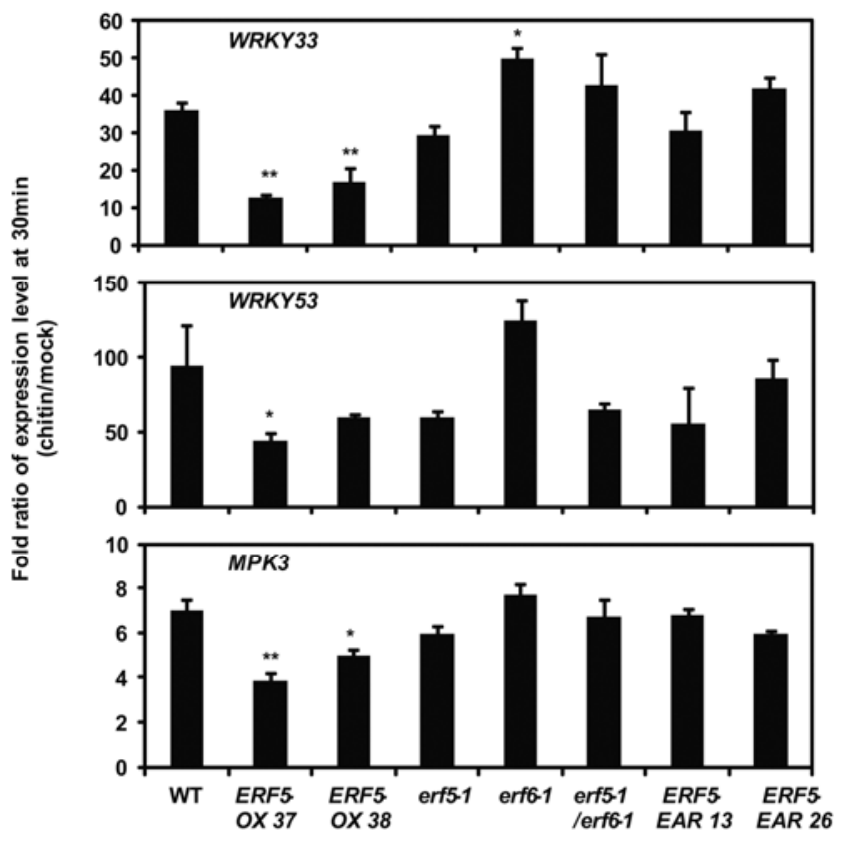

Fig. 6. Induction of chitin-responsive genes (CRG) by chitin in the wildtype (WT), ethylene-responsive element-binding factor (ERF)5-OX, erf51, erf6-1, erf5-1/erf6-1, and ERF5-EAR plants. Quantitative reverse-transcription polymerase chain reaction was employed to monitor CRG expression $30 \mathrm{~min}$ after treatment with chitin (chitooctaose, at a final concentration of $1 \mu \mathrm{M}$ ). Relative expression was calculated from the comparison with the mock-treated sample, with expression normalized to the internal control gene $S A N D$ in each sample. Average values of the results from three independent experiments were presented in the figure. Error bar, standard error. A statistically significant difference compared with the correspondingly treated WT plants was indicated with * $(P<0.05)$ or $* *(P<$ 0.01 ) based on Student's $t$ test. 
swer this question, we compared the response of wild-type, ERF5-OX (37 and 38), erf5-1, erf6-1, erf5-1/erf6-1, and ERF5$E A R$ (13 and 26) plants to inoculation with the bacterial pathogen $P$. syringae pv. tomato DC3000. Three days after infiltration with the bacterium ERF5-OX (37 and 38), plants showed significantly enhanced resistance to the pathogen compared with the wild type (Fig. 8). The erf5-1 plants supported a level of bacterial growth similar to that seen with wild-type plants (Fig. 8). However, the erf6-1 and the double mutant erf5-1/ erf6- 1 showed a slightly enhanced susceptibility to the pathogen. Similarly, the ERF5-EAR plants also showed enhanced susceptibility to the pathogen (Fig. 8). Together, these data suggest that ERF5, probably also ERF6, positively regulates plant defense against the biotrophic bacterial pathogen DC3000. Therefore, ERF5 appears to play opposing roles in plant defense: negatively regulating plant defense against necrotrophic fungal pathogens and positively regulating plant defense against biotrophic bacterial pathogens.

The data in Figure 8 also suggest that ERF5 and ERF6 are, to some degree, functionally redundant, in that the double mutant (erf5-1/erf6-1) was more susceptible to the pathogen than either of the single mutants. The slightly enhanced susceptibility of the ERF5-EAR plants to the bacterial pathogen is likely due to the strong suppression of the potential target genes of ERF5 by the EAR motif and, therefore, indirectly suggests a role of these target genes in plant defense against biotrophic bacterial pathogens.

\section{Expression of SA- and JA/ETH-pathway genes in wild-type, ERF5-OX, erf5-1, erf6-1, erf5-1/erf6-1, and $E R F 5-E A R$ plants.}

The opposing roles played by ERF5 in regulating plant defense against necrotrophic and biotrophic pathogens suggest that ERF5 may differentially regulate the SA- and JA/Ethpathways, because increasing evidence suggests that SA- and JA/ETH-pathways are antagonistic and play opposite roles in regulating plant defense to necrotrophic and biotrophic pathogens (Kachroo and Kachroo 2007; Kunkel and Brooks 2002; Li et al. 2004, 2006; Spoel et al. 2003; Thomma et al. 1998). To test this, we examined the expression of the SA-pathway marker genes $P R-1$ and $P R-2$, as well as SID2 (encoding an enzyme in the SA biosynthesis) and JA/ETH-pathway marker gene PDF1.2a (Thomma et al. 1998), as well as LOX2 (encoding an enzyme involved in the JA biosynthesis) and ACO2 (encoding an enzyme involved in the ETH biosynthesis) in the wild-type, ERF5-OX (37 and 38), erf5-1, erf6-1, erf5-1/erf6-1, and ERF5-EAR (13 and 26) plants. These comparisons showed that the expression of $P R-1, P R-2$, and SID2 was elevated in the EFR5-OX (37 and 38) plants compared with wild-type plants (Fig. 9). These data are consistent with the enhanced resistance of the ERF5-OX plants to the bacterial pathogen DC3000. The expression of these genes in erf5-1, erf6-1, erf51/erf6-1, and ERF5-EAR (13 and 26) plants appeared to be similar to that measured in wild-type plants (Fig. 9).

The expression of the JA/ETH-pathway gene ACO2 did not appear to be dramatically different in the wild-type, erf5-1, erf6-1, erf5-1/erf6-1, and ERF5-EAR (13 and 26) plants (Supplementary Fig. 7). However, the expression of $L O X 2$ was significantly suppressed in plants ectopically expressing ERF5, suggesting that ERF5 may negatively regulate the JA biosynthetic pathway.

Collectively, the data suggest that ERF5 may contribute to plant innate immunity to biotrophic pathogens by regulating SA signaling, while also affecting plant resistance to necrotrophic pathogens by regulating JA signaling. However, this is likely an oversimplification and not a complete depiction of

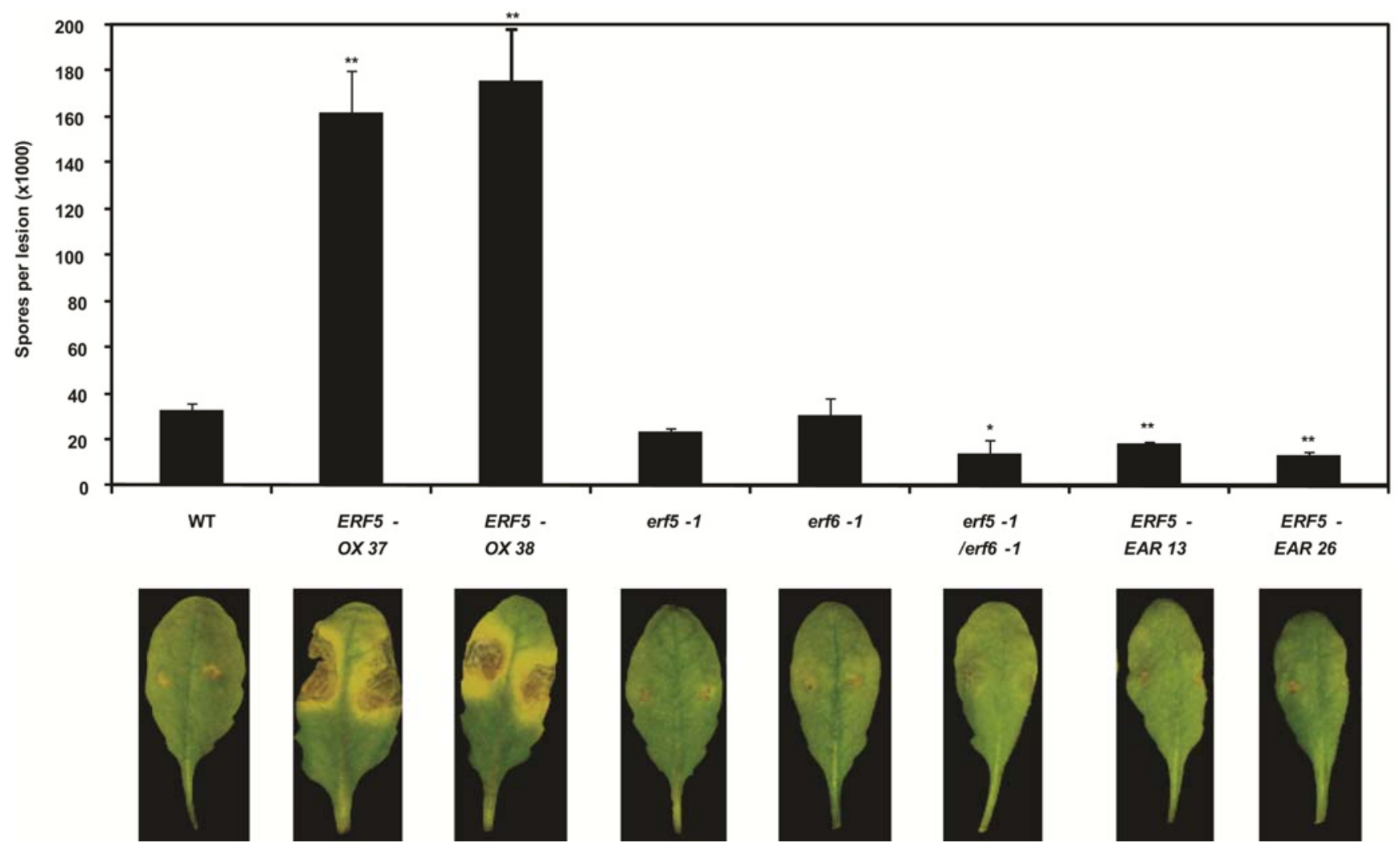

Fig. 7. Response of the wild-type (WT), ethylene-responsive element-binding factor (ERF)5-OX, erf5-1, erf6-1, erf5-1/erf6-1, and ERF5-EAR plants to a fungal pathogen. Spores of the fungal pathogen Alternaria brassicicola were spot inoculated onto the leaves of approximately 4-week-old plants at a concentration of $5 \times$ $10^{5}$ spores $/ \mathrm{ml}$. Average values of the results from three independent experiments were presented in the figure. Error bar, standard error. A statistically significant difference compared with the correspondingly treated WT plants was indicated with $*(P<0.05)$ or $* *(P<0.01)$ based on Student's $t$ test. 
the complex, interactive response networks that exist in plant cells.

Triple response of wild-type, $\mathrm{ERF5-OX}$, erf5-1, erf6-1, erf5-1/erf6-1, and ERF5-EAR plants.

In order to understand the potential mechanism underlining the regulatory role played by ERF5 (and also ERF6), we further tested the ETH-induced triple response of the wild-type, ERF5-OX (37 and 38), erf5-1, erf6-1, erf5-1/erf6-1, and ERF5$E A R$ (13 and 26) plants. These were obvious experiments because ERF5 is induced by ETH and encodes a member of the ERF family of TF proteins.

Plants were germinated in the presence of the ETH precursor 1-aminocyclopropane-1-carboxylic acid (ACC) in the dark. After 5 days of growth, the ERF5-OX plants were significantly more sensitive to ETH than wild-type plants. For example, the ERF5-OX plants produced shorter and thicker hypocotyls and shorter roots compared with the similarly treated wild-type plants. In contrast, the erf5-1/erf6-1 and ERF5-EAR plants appeared to be less sensitive to ETH, as reflected by the longer hypocotyls and roots, especially at the concentration of $0.5 \mu \mathrm{M}$ ACC, compared with the similarly treated wild-type plants (Fig. 10). The ETH-insensitive mutant ein2, included as a control, showed insensitivity to the ACC treatment, as previously demonstrated (Guzman and Ecker 1990). Because these plants appeared to germinate and grow similarly in the absence of ACC in the dark, the observed differences in the triple response by these plants is very likely caused by the manipulation of ERF5. Therefore, the data suggest that ERF5 affects plant sensitivity to ETH and is likely a positive regulator of ETH sig- naling in the triple response and other ETH-related processes as well. Because studies showed that ETH was involved in enhancing pathogen infection and symptom development associated with certain necrotrophic fungal pathogens (van Loon et al. 2006), the positive role of ERF5 in ETH signaling is consistent with the observed negative role of ERF5 in regulating plant defense against the necrotrophic fungal pathogen $A$. brassicicola (Fig. 7).

To help the reader follow these experiments, we have summarized all the results conducted on the wild-type, ERF5-OX (37 and 38), erf5-1, erf6-1, erf5-1/erf6-1, and ERF5-EAR (13 and 26) plants in Supplementary Table 5.

Regulation of ERF5 and ERF6 by chitin, SA, JA, and ETH.

The preceding experiments strongly suggest that ERF5 (and maybe ERF6) plays an important regulatory role in regulating chitin, SA, and ETH-signaling pathways. To further probe its potential relationship with these pathways, we examined the expression of ERF5 and ERF6 in wild-type plants at different time points after treatment with chitin (chitooctaose), SA, methyl jasmonic acid (MeJA), and ACC. In the case of chitooctaose treatment $(1 \mu \mathrm{M})$, we found that both genes, especially ERF5, were rapidly and strongly induced (Fig. 11A), consistent with our previous observations (Libault et al. 2007; Wan et al. $2008 \mathrm{~b})$. In the case of SA treatment $(500 \mu \mathrm{M})$, we found that both genes were also moderately induced by SA, with the induction of ERF6 being stronger (Fig. 11B). ERF6 also appeared to be moderately induced by MeJA $(10 \mu \mathrm{M})$; however, there was no significant effect on ERF5 expression by MeJA (Fig. 11C). In the above three experiments, the induction of

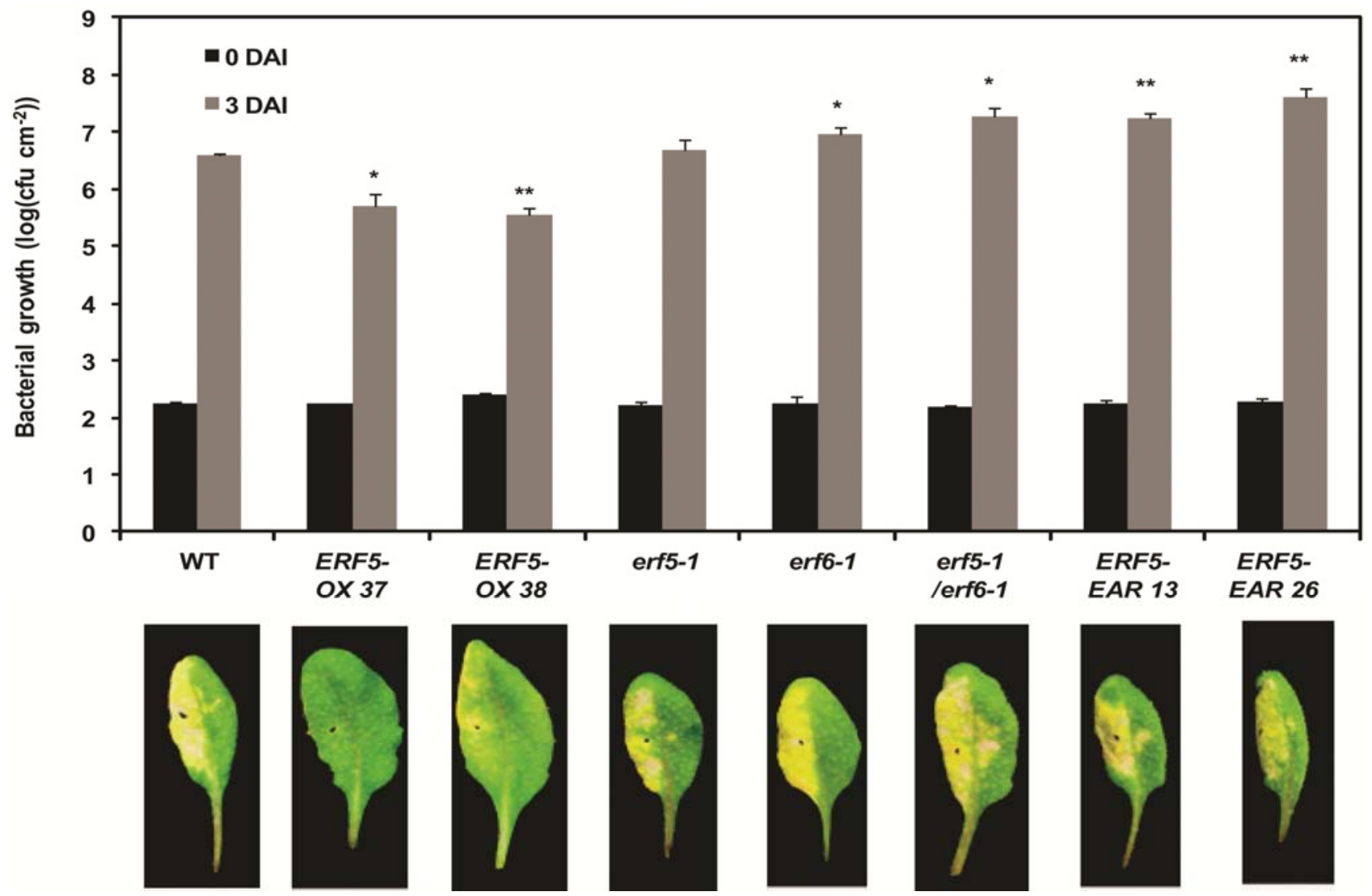

Fig. 8. Response of the wild-type (WT), ethylene-responsive element-binding factor (ERF)5-OX, erf5-1, erf6-1, erf5-1/erf6-1, and ERF5-EAR plants to a bacterial pathogen. In this experiment, Pseudomonas syringae pv. tomato DC3000 was infiltrated into the leaves of approximately 4-week-old plants at a concentration of $5 \times 10^{4} \mathrm{CFU} / \mathrm{ml}$. Bacterial growth rate was assayed at day 0 and 3, respectively. Average values of the results from three independent experiments were presented in the figure. Error bar, standard error. A statistically significant difference compared with the correspondingly treated WT plants was indicated with $*(P<0.05)$ or $* *(P<0.01)$ based on Student's $t$ test. 
ERF5 and ERF6 appeared to reach the maximum level approximately $30 \mathrm{~min}$ after the treatments. In the case of ACC treatment $(10 \mu \mathrm{M})$, both genes appeared to be slightly induced at $1 \mathrm{~h}$ after the treatment (Fig. 11D).

The induction of ERF5 and ERF6 by these different defense elicitors further supports their potential role in regulating different defense pathways in plants in response to different pathogens.

\section{DISCUSSION}

\section{Chitin-responsive TF and MPK appear}

to form a complex MPK/TF interaction network.

Our previous work showed that $118 \mathrm{TF}$ genes were regulated by chitin treatment (Libault et al. 2007), and MPK3 and MPK6 were activated by chitin (Wan et al. 2004). In this study, we found that many of these TF appeared to interact with other TF, as well as MPK3 and MPK6, suggesting that chitin signaling likely involves a complex interaction network. Studies of protein-protein interactions are dependent on the limitations of the available methods, all of which can be prone to artifactual results. Hence, it is important to confirm the results of one method using independent approaches. In the current study, initial results from the $\mathrm{Y} 2 \mathrm{H}$ screen were subsequently confirmed by pull-down experiments and in vitro kinase assays, as well as in planta BiFC experiments. Although each of these approaches can be criticized individually, collectively, they provide strong evidence that the interactions detected are real and can occur in planta. These findings are further confirmed by the fact that some of the interactions detected were also previously noted by other authors (e.g., WRKY40/WRKY18 and WRKY72/MPK6) (Popescu et al. 2008; Xu et al. 2006).

Although there is a tendency to draw linear models for plant signal transduction pathways, increasing evidence points to the actual involvement of complex networks in the control of plant gene expression and final cellular responses (Chory and $\mathrm{Wu}$ 2001; Eulgem 2006; Eulgem and Somssich 2007; Kendrick and Chang 2008; Uhrig 2006). The response of Arabidopsis to chitin elicitation resulted in the induction of 118 different $\mathrm{TF}$ genes (Libault et al. 2007) and approximately 800 other genes (Ramonell et al. 2005; Wan et al. 2008b). Our current data suggest that at least a subset of the proteins encoded by these TF genes function by direct protein-protein interactions to form a complex network to control downstream gene expression. Considering that only a subset of proteins encoded by the total 118 chitin-responsive TF genes was examined in detail in the current work, it is very likely that additional TF (as well as other regulatory proteins) may also be involved in this network. Therefore, we propose that P/MAMP signaling may involve a complex, multiprotein regulatory network that provides fine control over the plant response to pathogens and also serves to regulate such biotic stress responses with other growth parameters (e.g., those controlled by ETH signaling).

\section{Interactions between TF may confer specificity to regulation of downstream CRG.}

Many TF require a physical interaction with either an identical molecule or another similar TF protein to form a dimer to regulate the expression of their potential target genes. Such different combinations may specify the regulation of different genes and downstream events (Amoutzias et al. 2008). Interestingly, our present work revealed not only the interactions between members of the same TF family (e.g., ERF5/ERF6 and WRKY72/WRKY6) but also the interactions between members from two different families (e.g., SCL13/WRKY72, SCL13/ EFR5, and ERF5/WRKY33). Likely, the interactions between different TF may constitute another level of gene expression regulation. Our results are consistent with other literature reports. For example, Xu and colleagues (2006) recently showed that three WRKY TF (WRKY18, WRKY40, and WRKY60) interact to form both homodimers and heterodimers to create different DNA binding specificities. Therefore, the specificity of protein complex formation may potentially regulate the expression of different genes leading to distinct cellular responses (Xu et al. 2006). Therefore, future work should utilize the details of TF protein networks (Fig. 1) to explore how different TF complexes act to interpret cellular signaling to provide specificity.

\section{Phosphorylation of TF by MPK is likely involved in the regulation of chitin signaling.}

Wan and associates (2004) previously demonstrated the rapid activation of MPK3 and MPK6 in Arabidopsis by chitin (Wan et al. 2004). Our current work revealed that these MPK were capable of interacting with and phosphorylating various chitin-responsive TF and, by extension, potentially regulating downstream CRG expression. Indeed, one of the important roles of MPK is the regulation of gene expression through direct phosphorylation of TF (Chen and Thorner 2007; Rodriguez et al. 2010). Popescu and associates (2008) recently demonstrated that MPK3 and MPK6 were capable of phosphorylating a large number of proteins, including many TF, such as WRKY8, WRKY20, WRKY47, WRKY53, and WRKY69 (phosphorylated by MPK3); WRKY3, WRKY7, WRKY23, WRKY36, WRKY39, WRKY53, WRKY55, WRKY58, WRKY61, WRKY62, WRKY66, WRKY69, WRKY72, and WRKY75; and ERF6 (phosphorylated by MPK6) (Popescu et al. 2008). Recently, Mao and associates (2011) also showed that WRKY33 was phosphorylated by both MPK3 and MPK6. Among these, WRKY8, WRKY33, WRKY53, and WRKY72

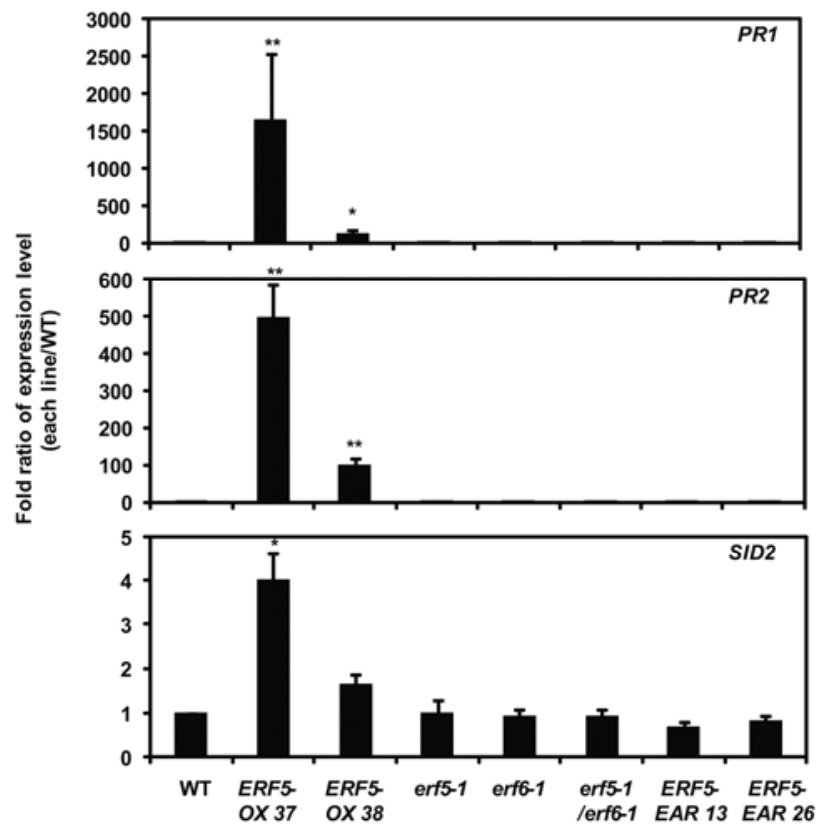

Fig. 9. Expression of salicylic acid (SA)-pathway genes in the wild-type (WT), ethylene-responsive element-binding factor (ERF)5-OX, erf5-1, erf6-1, erf5-1/erf6-1, and ERF5-EAR plants. Expression of SA-pathway genes $P R 1$ and $P R 2$ as well as $S I D 2$ was monitored using quantitative reverse-transcription polymerase chain reaction. Relative expression was calculated from the comparison with WT plants with each expression value normalized to the internal control gene $S A N D$ in each sample. Average values of the results from three independent experiments were presented in the figure. Error bar, standard error. A statistically significant difference compared with the correspondingly treated WT plants was indicated with $*(P<0.05)$ or $* *(P<0.01)$ based on Student's $t$ test. 
and ERF6 are also chitin responsive (Libault et al. 2007; Wan et al. 2004). Therefore, the regulation of gene expression by these MPK is likely very extensive and important in chitin signaling.
The MPK-TF interaction network is likely a key element in regulating plant defense.

Our current work suggests that the MPK/TF network revealed in this study is important in regulating plant defense. First of

A

$\begin{array}{lll}\text { ERF5 - } & \text { ERF5 - } & \text { erf5 -1 } \\ \text { OX } 37 & \text { ox } 38 & \end{array}$

erf6 -1

erf5 - $-1 /$

ERF5 -

ERF5 -

ein2
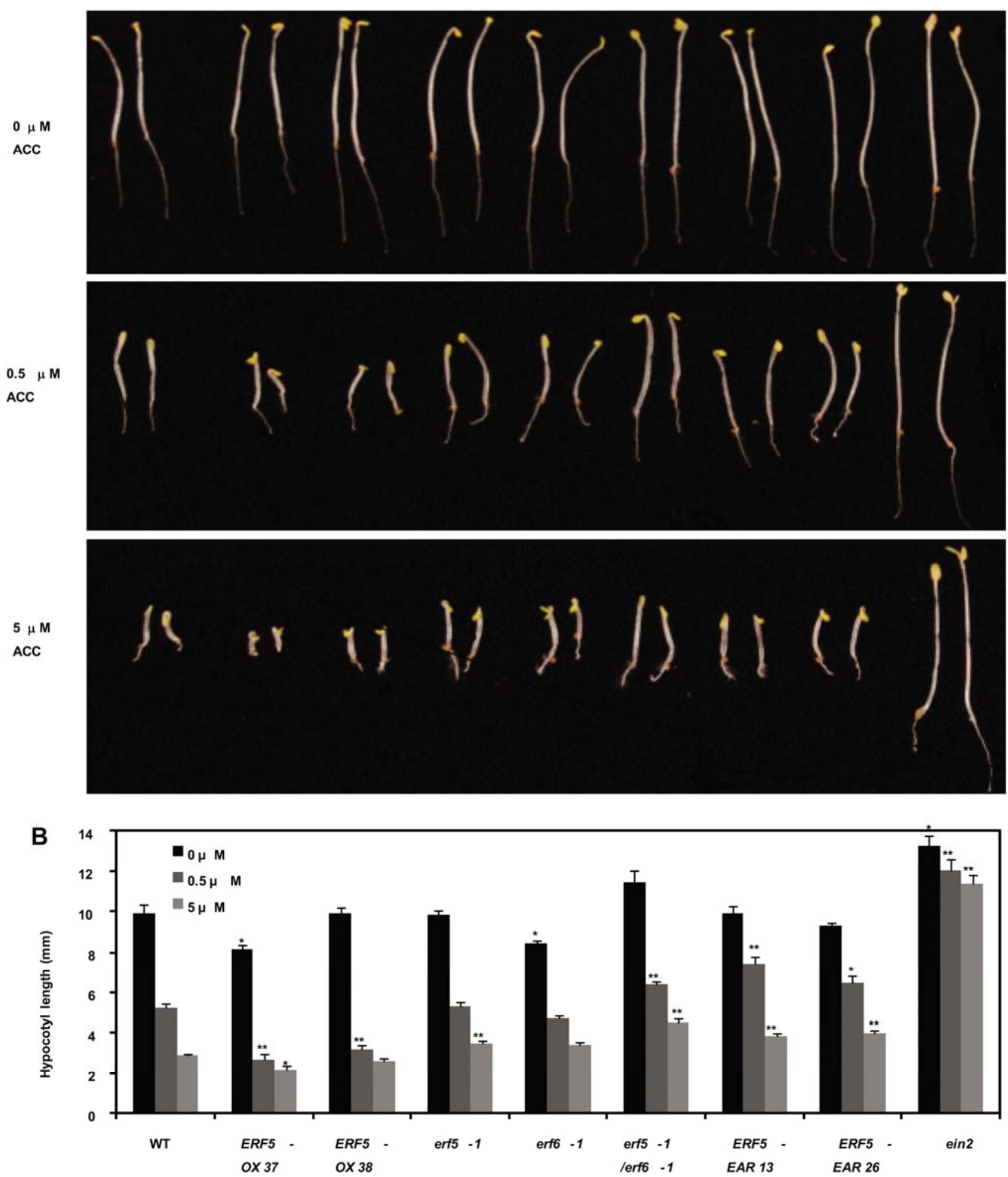

Fig. 10. Triple response of the wild-type (WT), ethylene-responsive element-binding factor (ERF)5-OX, erf5-1, erf6-1, erf5-1/erf6-1, and ERF5-EAR plants. Seed were germinated on the Murashige and Skoog medium in the presence of 1-aminocyclopropane-1-carboxylic acid (ACC) $(0,0.5$ and $5 \mu \mathrm{M})$ in the dark at $23^{\circ} \mathrm{C}$. Seedlings were photographed and measured 5 days later. A, Images of the 5-day old seedlings and $\mathbf{B}$, length of hypocotyls. Average length of 10 seedlings was presented with a standard error. A statistically significant difference compared with the correspondingly treated WT plants was indicated with $*(P<0.05)$ or ** $(P<0.01)$ based on Student's $t$ test. The experiment was repeated twice with similar results. 
all, every gene or protein in the network is either induced or activated by chitin (Libault et al. 2007; Ramonell et al. 2005; Wan et al. 2004, 2008b), and chitin is a P/MAMP which can activate plant defense (Wan et al. 2008a,b). Therefore, this network is likely important in regulating chitin-mediated plant defense. Second, the majority of the proteins in the network have a proven role in plant defense; for example, MPK3 and MPK6 (Pedley and Martin 2005; Zhang and Klessig 2001) and WRKY6, WRKY18, WRKY33, WRKY40, and WRKY72 (Andreasson et al. 2005; Bhattarai et al. 2010; Chen and Chen 2002; Lippok et al. 2007; Mao et al. 2011; Qiu et al. 2008; Robatzek and Somssich 2001, 2002; Xu et al. 2006; Zheng et al. 2006). Additionally, ERF5, ERF6, and ERF 8 are responsive to ETH and, therefore, are potentially important in regulating both ETH signaling and defense-related genes, such as PDF 1.2a, PR-2, PR-3, and PR-4 (Broekaert et al. 2006; Nakano et al. 2006). Third, our current work demonstrated the possible role of the key node protein, ERF5, as well as ERF6, in plant defense to pathogen infection.

ERF5 appears to play opposing roles in the control of plant defense in response to infection by a necrotrophic fungal pathogen or biotrophic bacterial pathogen.

Our data also showed that ectopic expression of ERF5 led to enhanced susceptibility to the necrotrophic fungal pathogen $A$. brassicicola. In contrast, the ERF5 mutation, especially in combination with the ERF6 mutation, and the suppression of the potential target genes of ERF5 through expression of the ERF5EAR construct led to enhanced resistance to the fungal pathogen. Meanwhile, in the case of infection with the biotrophic bacterial pathogen, ectopic expression of ERF5 led to enhanced resistance. In contrast, the ERF5 mutation, especially in combination with the ERF6 mutation, and the suppression of the potential target genes of ERF5 through expression of the ERF5-EAR construct led to enhanced susceptibility to the bacterial pathogen. Therefore, ERF5 likely plays opposing roles in regulating plant defense against these different pathogens. This is probably due to differential effects caused by ERF5 on the chitin and SA or ETH signaling pathways, because our data suggest that ERF5 may negatively regulate chitin-responsive genes and positively regulate SA-pathway genes. Additionally, the manipulation of the ERF5 expression or its potential target genes also affected the ETH-induced triple response, suggesting that ERF5 may also regulate the ETH signaling pathway. Therefore, ERF5 appears to be an important regulator to coordinate plant defense to different pathogens through differentially regulating different defense signaling pathways. It is our view that the integration of these signals, at least in part, is likely mediated by a complex, interactive TF network.

\section{MATERIALS AND METHODS}

Cloning.

The open reading frames (ORF) of chitin-inducible TF and MPK genes were amplified from Arabidopsis thaliana (Col-0) cDNA, and cloned into the vector pDONOR201 using BP clonase according to the GATEWAY cloning procedure (Invitrogen, Carlsbad, CA, U.S.A.). The cloned ORF were then moved into other destination vectors using LR clonase according to the GATEWAY cloning procedure (Invitrogen) for either $\mathrm{Y} 2 \mathrm{H}$ screenings or protein expression (discussed below).

\section{Y2H screens.}

The yeast strain PJ69-4a (MATa trp1-901 leu2-3,112 ura352 his3-200 gal4 [deleted] gal80 [deleted] LYS2::GAL1-HIS3 GAL2-ADE2 met2::GAL7-lacZ) was used for Y2H screens. Each TF ORF was mobilized from pDONOR201 into pDEST22 (as the prey) or pDEST32 (as the bait) using the Gateway cloning procedure (Invitrogen). Each pair of the bait and prey constructs was transformed into PJ69-4a by a standard yeast transformation procedure (Clontech, Mountain View, CA, U.S.A.) and the transformation mixture was spotted on SD media (-Trp-Leu, -Trip-Leu-His [0.1 mM, 0.5 mM, or $1 \mathrm{mM}$ 3-amino-1,2,4-triazole (3-AT)], -Trp-Leu-Ade-His). 3-AT is a
A
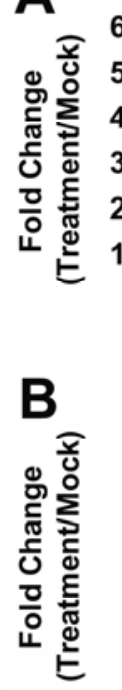

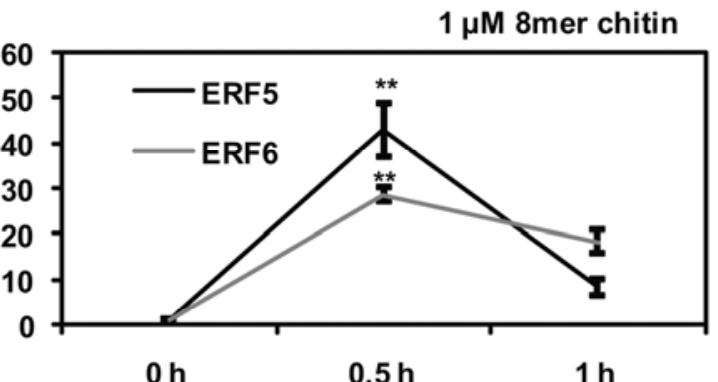

$500 \mu \mathrm{M}$ SA

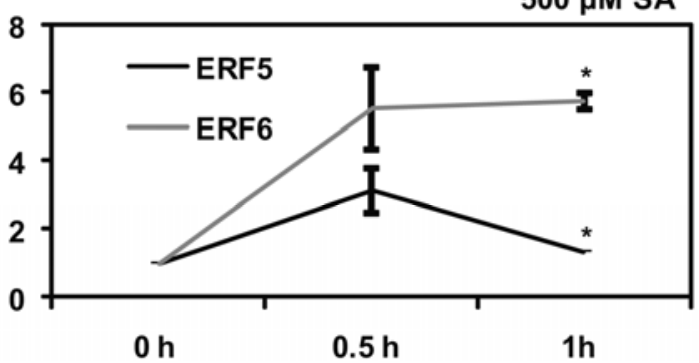

C
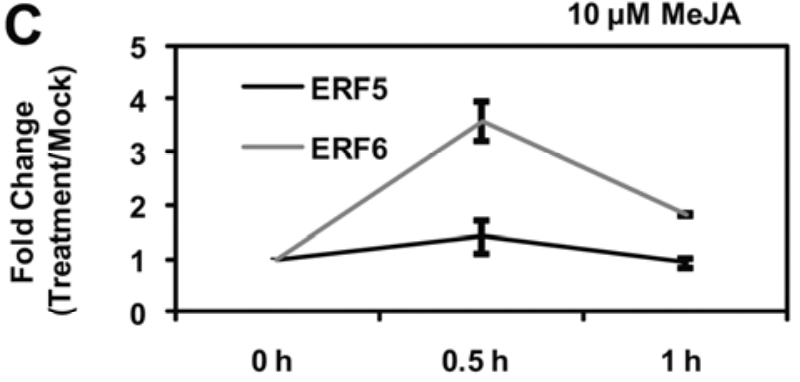

$10 \mu \mathrm{M}$ ACC

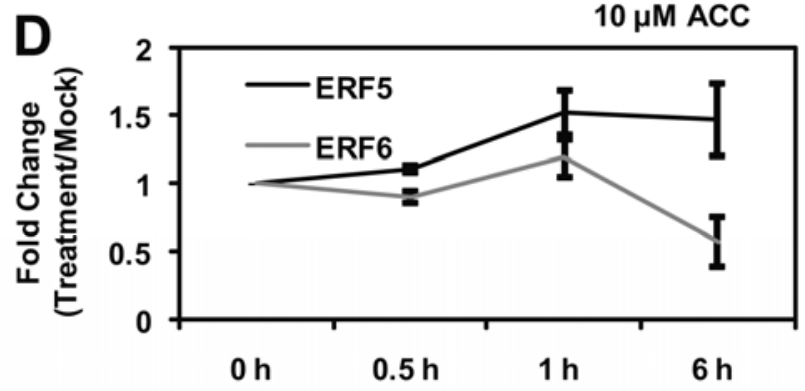

Fig. 11. Expression of ethylene-responsive element-binding factor $(E R F) 5$ and $E R F 6$ in response to chitin, salicylic acid (SA), jasmonic acid (JA), and ethylene (ETH). Quantitative reverse-transcription polymerase chain reaction was employed here to measure the expression of these genes in wild-type IWT) plants at 0, 30, and 60 min after treatment with A, chitin; B, SA; C, methyl jasmonic acid (MeJA); and D, 1-aminocyclopropane-1-carboxylic acid (ACC) (the precursor of ETH), respectively. Relative expression was calculated from the comparison with the mock-treated WT plants with each expression value normalized to the internal control gene $S A N D$ in each sample. Average values of the results from two independent experiments were presented in the figure. Error bar, standard error. A statistically significant difference compared with the correspondingly treated WT plants was indicated with $*(P<0.05)$ or $* *(P<$ 0.01 ) based on Student's $t$ test. 
competitive inhibitor of the product (histidine or His) of the HIS3 gene. Plates were incubated at $30^{\circ} \mathrm{C}$ and were examined 4 days later.

\section{In vitro pull-down assay.}

To make tagged TF proteins, the ORF of ERF5, ERF6, ERF8, WRKY6, WRKY18, WRKY40, WRKY72, and SCL13 in the gateway entry vector pDONOR201 were subcloned into pDEST15 (GST-tagged vector; Invitrogen) and pDEST17 (Histagged vector; Invitrogen) via LR cloning reactions (Invitrogen). Each construct was transformed into $E$. coli strain BL21-AI (F rec $\mathrm{A} 1$ end $\mathrm{A} 1$ hsd $\mathrm{R} 17\left[\mathrm{r}_{\mathrm{k}}^{-}, \mathrm{m}_{\mathrm{k}}^{+}\right]$sup $\mathrm{E} 44 \lambda^{-}$thi-1 gyrA96 relA1) (Invitrogen) for protein expression, and the expression of recombinant proteins was induced by L(+)-arabinose. GST- and Histagged proteins were extracted in the co-immunoprecipitation (Co-IP) buffer (100 mM Tris-HCl [pH 8.0], $150 \mathrm{mM} \mathrm{NaCl,}$ $0.5 \%$ NP-40, $1 \mathrm{mM}$ EDTA]. GST-tagged protein extracts were incubated with prewashed $50 \%$ gluthathione sepharose beads (Peptron, Yuseong-Gu, Daejeon, South Korea) and the beads were then washed five times with Co-IP buffer. To test the in vitro interaction between two proteins, a purified GST-tagged protein with beads was incubated with a His-tagged protein lysate in $1 \times$ binding buffer $(50 \mathrm{mM}$ Tris- $\mathrm{HCl}$ [pH 7.5], $150 \mathrm{mM}$ $\mathrm{NaCl}, 0.5 \%$ NP-40, 2 mM EGTA, 10 mM EDTA, 1 mM dithiothreitol [DTT], $1 \mathrm{mM}$ PMSF) for $4 \mathrm{~h}$ at $4^{\circ} \mathrm{C}$ on a rotator and then the precipitated beads were washed five times with $1 \times$ binding buffer without DTT. The co-precipitated proteins were separated on a $12.5 \%$ sodium dodecyl sulfate (SDS)-polyacrylamide gel and detected by an anti-His antibody (Amersham Biosciences, Piscataway, NJ, U.S.A.), respectively.

\section{BiFC.}

Each BiFC construct was electroporated into Agrobacterium tumefaciens GV3101. Different Agrobacterium strains were cultured overnight, pelleted, and resuspended in $10 \mathrm{mM} \mathrm{MgCl}_{2}$, including $40 \mu \mathrm{M}$ acetosyringone (Sigma-Aldrich, St. Louis). After pretreatment with acetosyringone for approximately $2 \mathrm{~h}$ at room temperature, the Agrobacterium strains harboring the two different $\mathrm{BiFC}$ constructs were mixed with the third A. tumefaciens (C58C1) expressing the silencing suppressor HC-Pro (Kwon et al. 2009), with each strain adjusted to a final optical density of 0.3 at $600 \mathrm{~nm}$. The bacterial mixture was co-infiltrated into leaves of 4-week-old $N$. benthamiana plants using a syringe, to examine protein-protein interactions. The same construct pair was infiltrated into multiple locations on different leaves. Three days after infiltration, the infiltrated area was cut and observed using a confocal microscope (a Zeiss LSM 510 META NLO two-photon point-scanning confocal system).

\section{In vitro kinase assay.}

Recombinant His-tagged MPK were purified using nickel columns (Qiagen, Valencia, CA, U.S.A.) and used to phosphorylate recombinant GST-tagged substrate proteins. GSTtagged proteins were purified using glutathione-sepharose 4B columns (GE Healthcare, Piscataway, NJ, U.S.A.). Purified MPK (200 ng) and $500 \mathrm{ng}$ of each substrate protein were incubated in $20 \mu$ of kinase reaction buffer $(50 \mathrm{mM}$ Tris- $\mathrm{HCl}[\mathrm{pH}$ 7.5], $10 \mathrm{mM} \mathrm{MgCl}$, and $10 \mu \mathrm{Ci}$ of $\gamma^{-32} \mathrm{P}$-ATP) at $30^{\circ} \mathrm{C}$ for 30 min. The phosphorylation reactions were stopped by the addition of SDS-loading buffer. The phosphorylated substrates were visualized by autoradiography after being separated in a $12.5 \%$ SDS-polyacrylamide gel.

\section{Genotyping of insertion mutants.}

The ERF5 insertion mutant 681E07 (named erf5-1) was generated in the context of the GABI-Kat program and provided by Bernd Weisshaar (Max Planck Institute for Plant
Breeding Research, Cologne, Germany) (Rosso et al. 2003). The ERF6 insertion mutant SALK_087356 (named erf6-1) was generated by the SALK Institute (Alonso et al. 2003b) and distributed through the Arabidopsis Biological Resource Center. The homozygous erf5-1 plants were identified by genotyping using the following gene-specific primers: 5'-ATGGCGA CTCCTAACGAAGTA-3' (forward) and 5'-AACAACGGTC AACTGGGAATA-3' (reverse), together with the T-DNA left border primer 5'-CCCATTTGGACGTGAATGTAGACAC-3'; the homozygous erf6-1 plants were identified by genotyping using the following gene-specific primers: 5'-ATGGCTACAC CAAACGAAGTA-3' (forward) and 5'-AACAACGGTCAATT GTGGATA-3' (reverse), together with the T-DNA left border primer 5'-GCGTGGACCGCTTGCTGCAACT-3'.

\section{Making of the CaMV35S ERF5 and $E R F 5-E A R$ fusion constructs.}

The gateway cartridge was mobilized into the HA-pBA vector (a gift from H. S. Seo, Seoul National University, Korea) from pMDC43 (Curtis and Grossniklaus 2003) with the following forward and reverse primers: 5'-TTAATTAAAGGCGC GCCAAGCTATCAAACAAG-3' and 5'-CGGCCGCTCTAGA ACTAGTTAATTAA-3'. The ERF5 ORF was then inserted from the entry clone into the above-modified HA-pBA gateway vector by LR reaction. Additionally, the ERF5 ORF was also cloned into $\mathrm{pH} 35 \mathrm{GEAR}$ by LR reaction. The $\mathrm{pH} 35 \mathrm{GEAR}$ was a gift from T. Demura (Kubo et al. 2005) to make the ERF5-EAR fusion construct. The final constructs were electroporated into A. tumefaciens to transform Arabidopsis thaliana using the floral dip approach (Clough and Bent 1998). The T3 homozygous lines were identified by the 3:1 segregation of T2 plants under antibiotic selection.

\section{Growth of seedlings and treatment with chitin or other chemicals.}

Arabidopsis seedlings were grown in half-strength Murashige and Skoog (MS) liquid medium as described (Zhang et al. 2002). Seedlings, approximately 10 days old, were treated with chitooctaose (8 mer; stock solution prepared in water), SA (stock solution prepared in ethanol), MeJA (stock solution prepared in ethanol), and ACC (stock solution prepared in water) at a final concentration of $1,500,10$, and $10 \mu \mathrm{M}$, respectively, for the time indicated in each related figure. All these chemicals were purchased from Sigma-Aldrich. The control plants were similarly treated with an equivalent amount of distilled water or ethanol. After treatment, the seedlings were collected and frozen in liquid nitrogen for RNA isolation.

\section{ETH-induced triple response assay.}

The triple response assay was performed as described (Alonso et al. 2003a). Briefly, seed were sterilized and germinated on the following medium: $1 \times$ MS salts (Sigma-Aldrich), $1 \%$ sucrose, and $0.8 \%$ agar $(\mathrm{pH} \mathrm{5.7)}$, in the presence of ACC $(0$, $0.5 \mathrm{~m}$ and $5 \mu \mathrm{M}$ ) (Sigma-Aldrich), in the dark at $23^{\circ} \mathrm{C}$. The seedlings were photographed and measured 5 days later.

\section{RNA isolation and cDNA synthesis.}

Total RNA was isolated using the Trizol Reagent according to the manufacturer's instructions (Invitrogen). The isolated RNA was further treated with Turbo DNase (Ambion, Austin, TX, U.S.A.). cDNA was synthesized using M-MLV reverse transcriptase according to the manufacturer's instructions (Promega Corp., Madison, WI, U.S.A.).

\section{RT-PCR.}

The gene-specific primer pairs (forward and reverse) for detecting ERR5 and ERR6 were 5'-AGCTAAAACAGAGTGG 
ATTCA-3' and 5'-AACAACGGTCAACTGGGAATA-3' for ERF5 (At5g47230) and 5'-TCCTCGCGTTACTGTTCAAT$3^{\prime}$ and 5'-AACAACGGTCAATTGTGGATA-3' for ERF6 (At4g17490). As an internal control, the following forward and reverse primers of actin-2 (At3g18780) were used: 5'-GAC TAAGAGAGAAAGTAAGAGATAATCCAG-3' and 5'-CAGC CTTTGATTTCAATTTGCATGTAAGAG-3'. To analyze gene expression using RT-PCR, equivalent amounts of cDNA were included in PCR reactions which contained both a gene-specific primer pair and the actin-2 primer pair under the following PCR conditions: $94^{\circ} \mathrm{C}$ for $5 \mathrm{~min} ; 29$ cycles of $94^{\circ} \mathrm{C}$ for 40 $\mathrm{s}, 55^{\circ} \mathrm{C}$ for $40 \mathrm{~s}$, and $72^{\circ} \mathrm{C}$ for $2 \mathrm{~min}$; concluding with $72^{\circ} \mathrm{C}$ for 5 min. The resultant PCR products were resolved on a $1.2 \%$ agarose gel for comparison. The actin-2 band served as an internal control.

\section{qRT-PCR.}

The expression of the following genes was quantified by qRT-PCR using the primers listed in Supplementary Table 6 and in references (Kwon et al. 2009; Libault et al. 2007): ERF5, ERF6, MPK3, SID2, LOX2, ACO2, WRKY33, WRKY53, $P R-1, P R-2, P D F 1.2 a$, and SAND. The SAND gene was used as an internal control to normalize gene expression across different samples. The reactions were conducted on a 7500 RealTime PCR System (Applied Biosystems, Foster City, CA, U.S.A.) using the SYBR Green Master Mix (Applied Biosystems) with the following conditions: $95^{\circ} \mathrm{C}$ for $10 \mathrm{~min}$ and 40 cycles of $95^{\circ} \mathrm{C}$ for $15 \mathrm{~s}$ and $60^{\circ} \mathrm{C}$ for $1 \mathrm{~min}$, followed by the dissociation curve analysis to verify single amplicons. The relative fold change of the target gene, normalized to At2g28390 and relative to the gene expression in the control sample, was calculated as described (Libault et al. 2007).

\section{Disease assays.}

The disease assay with Alternaria brassicicola was conducted as described (Veronese et al. 2006) with a spore suspension of $5 \times 10^{5}$ spores $/ \mathrm{ml}$ by dot inoculating $5 \mu \mathrm{l}$ of the spore solution. The disease assays with $P$. syringae pv. tomato DC3000 was conducted as previously described (Kwon et al. 2009) with a bacterial concentration of $5 \times 10^{4} \mathrm{CFU} / \mathrm{ml}$.

\section{ACKNOWLEDGMENTS}

We thank the Arabidopsis Biological Resource Center and the GABIKAT (MPI for Plant Breeding Research, Cologne, Germany) for the mutants used in the current work, C. Lawrence for Alternaria brassicicola, S. Gelvin for the BiFC vectors, S. Bhattacharjee for help with the BiFC experiments, Mr. R. Zhang for assistance with plant growth and seed harvesting, J. Wang for tobacco plants, and all the reviewers and editors for their time and efforts in reviewing the manuscript and providing very constructive suggestions. The work was supported by a grant from the Unites States Department of Energy (DE-FG02-08ER15309) to G. Stacey, Basic Science Research Program through the National Research Foundation of Korea (2007-0053802 and 2010-0012801) and a grant from teh Next Generation Biogreen 21 Program (Systems \& Synthetic Agrobiotech Center grant PJ008173) to J. C. Hong, and the World Class University Program (R32-10148) funded by MOEST to W. S. Chung. G. H. Son was supported by scholarships from the BK21 program funded by MOEST.

\section{LITERATURE CITED}

Agarwal, P. K., Agarwal, P., Reddy, M. K., and Sopory, S. K. 2006. Role of DREB transcription factors in abiotic and biotic stress tolerance in plants. Plant Cell Rep 25:1263-1274.

Alonso, J. M., Stepanova, A. N., Leisse, T. J., Kim, C. J., Chen, H., Shinn, P., Stevenson, D. K., Zimmerman, J., Barajas, P., Cheuk, R., Gadrinab, C., Heller, C., Jeske, A., Koesema, E., Meyers, C. C., Parker, H., Prednis, L., Ansari, Y., Choy, N., Deen, H., Geralt, M., Hazari, N., Hom, E., Karnes, M., Mulholland, C., Ndubaku, R., Schmidt, I., Guzman, P., Aguilar-Henonin, L., Schmid, M., Weigel, D., Carter, D. E., Marchand,
T., Risseeuw, E., Brogden, D., Zeko, A., Crosby, W. L., Berry, C. C., Ecker, J. R. 2003a. Genome-wide insertional mutagenesis of Arabidopsis thaliana. Science 301:653-657.

Alonso, J. M., Stepanova, A. N., Solano, R., Wisman, E., Ferrari, S., Ausubel, F. M., and Ecker, J. R. 2003b. Five components of the ethyleneresponse pathway identified in a screen for weak ethylene-insensitive mutants in Arabidopsis. Proc. Natl. Acad. Sci. U.S.A. 100:2992-2997.

Amoutzias, G. D., Robertson, D. L., Van de Peer, Y., and Oliver, S. G. 2008. Choose your partners: Dimerization in eukaryotic transcription factors. Trends Biochem. Sci. 33:220-229.

Andreasson, E., Jenkins, T., Brodersen, P., Thorgrimsen, S., Petersen, N. H., Zhu, S., Qiu, J. L., Micheelsen, P., Rocher, A., Petersen, M., Newman, M. A., Bjorn Nielsen, H., Hirt, H., Somssich, I., Mattsson, O., and Mundy, J. 2005. The MAP kinase substrate MKS1 is a regulator of plant defense responses. EMBO (Eur. Mol. Biol. Organ.) J. 24:2579-2589.

Asai, T., Tena, G., Plotnikova, J., Willmann, M. R., Chiu, W. L., GomezGomez, L., Boller, T., Ausubel, F. M., and Sheen, J. 2002. MAP kinase signalling cascade in Arabidopsis innate immunity. Nature 415:977983.

Bader, S., Kuhner, S., and Gavin, A. C. 2008. Interaction networks for systems biology. FEBS (Fed. Eur. Biochem. Soc.) Lett. 582:1220-1224.

Bhattacharjee, S., Lee, L.Y., Oltmanns, H., Cao, H., Veena, Cuperus, J., and Gelvin, S. B. 2008. IMPa-4, an Arabidopsis importin alpha isoform, is preferentially involved in agrobacterium-mediated plant transformation. Plant Cell 20:2661-2680.

Bhattarai, K. K., Atamian, H. S., Kaloshian, I., and Eulgem, T. 2010. WRKY72-type transcription factors contribute to basal immunity in tomato and Arabidopsis as well as gene-for-gene resistance mediated by the tomato R gene Mi-1. Plant J. 63:229-240.

Broekaert, W. F., Delaure, S. L., De Bolle, M. F., and Cammue, B. P. 2006. The role of ethylene in host-pathogen interactions. Annu. Rev. Phytopathol. 44:393-416.

Chen, C., and Chen, Z. 2002. Potentiation of developmentally regulated plant defense response by AtWRKY18, a pathogen-induced Arabidopsis transcription factor. Plant Physiol. 129:706-716.

Chen, R. E., and Thorner, J. 2007. Function and regulation in MAPK signaling pathways: Lessons learned from the yeast Saccharomyces cerevisiae. Biochim. Biophys. Acta 1773:1311-1340.

Chory, J., and Wu, D. 2001. Weaving the complex web of signal transduction. Plant Physiol. 125:77-80.

Ciftci-Yilmaz, S., and Mittler, R. 2008. The zinc finger network of plants. Cell Mol. Life Sci. 65:1150-1160.

Citovsky, V., Lee, L. Y., Vyas, S., Glick, E., Chen, M. H., Vainstein, A., Gafni, Y., Gelvin, S. B., and Tzfira, T. 2006. Subcellular localization of interacting proteins by bimolecular fluorescence complementation in planta. J. Mol. Biol. 362:1120-1131.

Clough, S. J., and Bent, A. F. 1998. Floral dip: A simplified method for Agrobacterium-mediated transformation of Arabidopsis thaliana. Plant J. 16:735-743.

Curtis, M. D., and Grossniklaus, U. 2003. A gateway cloning vector set for high-throughput functional analysis of genes in planta. Plant Physiol. 133:462-469.

Eulgem, T. 2006. Dissecting the WRKY web of plant defense regulators. PLoS Pathog. 2:1028-1030.

Eulgem, T., and Somssich, I. E. 2007. Networks of WRKY transcription factors in defense signaling. Curr. Opin. Plant Biol. 10:366-371.

Fujimoto, S.Y., Ohta, M., Usui, A., Shinshi, H., and Ohme-Takagi, M. 2000. Arabidopsis ethylene-responsive element binding factors act as transcriptional activators or repressors of GCC box-mediated gene expression. Plant Cell 12:393-404.

Grove, C. A., and Walhout, A. J. 2008. Transcription factor functionality and transcription regulatory networks. Mol. Biosyst. 4:309-314.

Guzman, P., and Ecker, J. R. 1990. Exploiting the triple response of Arabidopsis to identify ethylene-related mutants. Plant Cell 2:513-523.

Guzman-Vargas, L., and Santillan, M. 2008. Comparative analysis of the transcription-factor gene regulatory networks of $E$. coli and S. cerevisiae. BMC Syst. Biol. 2:13-22.

Hiratsu, K., Matsui, K., Koyama, T., and Ohme-Takagi, M. 2003. Dominant repression of target genes by chimeric repressors that include the EAR motif, a repression domain, in Arabidopsis. Plant J. 34:733-739.

Iizasa, E. I., Mitsutomi, M., and Nagano, Y. 2009. Direct binding of a plant LysM receptor-like kinase, LysM RLK1/CERK1, to chitin in vitro. J. Biol. Chem. 285:2996-3004.

Kachroo, A., and Kachroo, P. 2007. Salicylic acid-, jasmonic acid- and ethylene-mediated regulation of plant defense signaling. Genet. Eng. (NY) 28:55-83.

Kaku, H., Nishizawa, Y., Ishii-Minami, N., Akimoto-Tomiyama, C., Dohmae, N., Takio, K., Minami, E., and Shibuya, N. 2006. Plant cells recognize chitin fragments for defense signaling through a plasma membrane receptor. Proc. Natl. Acad. Sci. U.S.A. 103:11086-11091. 
Kendrick, M. D., and Chang, C. 2008. Ethylene signaling: New levels of complexity and regulation. Curr. Opin. Plant Biol. 11:479-485.

Kerppola, T. K. 2008. Bimolecular fluorescence complementation (BiFC) analysis as a probe of protein interactions in living cells. Annu. Rev. Biophys. 37:465-487.

Kerppola, T. K. 2009. Visualization of molecular interactions using bimolecular fluorescence complementation analysis: Characteristics of protein fragment complementation. Chem. Soc. Rev. 38:2876-2886.

Kubo, M., Udagawa, M., Nishikubo, N., Horiguchi, G., Yamaguchi, M. Ito, J., Mimura, T., Fukuda, H., and Demura, T. 2005. Transcription switches for protoxylem and metaxylem vessel formation. Genes Dev. 19:1855-1860.

Kunkel, B. N., and Brooks, D. M. 2002. Cross talk between signaling pathways in pathogen defense. Curr. Opin. Plant Biol. 5:325-331.

Kwon, S. I., Kim, S. H., Bhattacharjee, S., Noh, J. J., and Gassmann, W. 2009. SRFR1, a suppressor of effector-triggered immunity, encodes a conserved tetratricopeptide repeat protein with similarity to transcriptional repressors. Plant J. 57:109-119.

Li, J., Brader, G., and Palva, E. T. 2004. The WRKY70 transcription factor: A node of convergence for jasmonate-mediated and salicylate-mediated signals in plant defense. Plant Cell 16:319-331.

Li, J., Brader, G., Kariola, T., and Palva, E. T. 2006. WRKY70 modulates the selection of signaling pathways in plant defense. Plant J. 46:477-491.

Libault, M., Wan, J., Czechowski, T., Udvardi, M., and Stacey, G. 2007. Identification of 118 Arabidopsis transcription factor and 30 ubiquitinligase genes responding to chitin, a plant-defense elicitor. Mol. PlantMicrobe Interact. 20:900-911.

Lippok, B., Birkenbihl, R. P., Rivory, G., Brummer, J., Schmelzer, E., Logemann, E., and Somssich, I. E. 2007. Expression of AtWRKY33 encoding a pathogen- or PAMP-responsive WRKY transcription factor is regulated by a composite DNA motif containing W box elements. Mol. Plant-Microbe Interact. 20:420-429.

Mao, G., Meng, X., Liu, Y., Zheng, Z., Chen, Z., and Zhang, S. 2011. Phosphorylation of a WRKY transcription factor by two pathogenresponsive MAPKs drives phytoalexin biosynthesis in Arabidopsis. Plant Cell 23:1639-1653.

Miya, A., Albert, P., Shinya, T., Desaki, Y., Ichimura, K., Shirasu, K., Narusaka, Y., Kawakami, N., Kaku, H., and Shibuya, N. 2007. CERK1, a LysM receptor kinase, is essential for chitin elicitor signaling in Arabidopsis. Proc. Natl. Acad. Sci. U.S.A. 104:19613-19618.

Nakano, T., Suzuki, K., Fujimura, T., and Shinshi, H. 2006. Genome-wide analysis of the ERF gene family in Arabidopsis and rice. Plant Physiol. 140:411-432.

Obayashi, T., Kinoshita, K., Nakai, K., Shibaoka, M., Hayashi, S., Saeki, M., Shibata, D., Saito, K., and Ohta, H. 2007. ATTED-II: A database of co-expressed genes and cis elements for identifying co-regulated gene groups in Arabidopsis. Nucleic Acids Res. 35:D863-D869.

Obayashi, T., Hayashi, S., Saeki, M., Ohta, H., and Kinoshita, K. 2009. ATTED-II provides coexpressed gene networks for Arabidopsis. Nucleic Acids Res. 37:D987-D991.

Ohta, M., Matsui, K., Hiratsu, K., Shinshi, H., and Ohme-Takagi, M. 2001. Repression domains of class II ERF transcriptional repressors share an essential motif for active repression. Plant Cell 13:1959-1968.

Pandey, S. P., and Somssich, I. E. 2009. The role of WRKY transcription factors in plant immunity. Plant Physiol. 150:1648-1655.

Pearson, R. B., and Kemp, B. E. 1991. Protein kinase phosphorylation site sequences and consensus specificity motifs: Tabulations. Methods Enzymol. 200:62-81

Pedley, K. F., and Martin, G. B. 2005. Role of mitogen-activated protein kinases in plant immunity. Curr. Opin. Plant Biol. 8:541-547.

Popescu, S. C., Popescu, G. V., Bachan, S., Zhang, Z., Gerstein, M., Snyder, M., and Dinesh-Kumar, S. P. 2008. MAPK target networks in Arabidopsis thaliana revealed using functional protein microarrays. Genes Dev. 23:80-92.

Pre, M., Atallah, M., Champion, A., De Vos, M., Pieterse, C. M., and Memelink, J. 2008. The AP2/ERF domain transcription factor ORA59 integrates jasmonic acid and ethylene signals in plant defense. Plant Physiol. 147:1347-1357.

Qiu, J. L., Fiil, B. K., Petersen, K., Nielsen, H. B., Botanga, C. J., Thorgrimsen, S., Palma, K., Suarez-Rodriguez, M. C., SandbechClausen, S., Lichota, J., Brodersen, P., Grasser, K. D., Mattsson, O., Glazebrook, J., Mundy, J., and Petersen, M. 2008. Arabidopsis MAP kinase 4 regulates gene expression through transcription factor release in the nucleus. EMBO (Eur. Mol. Biol. Organ.) J. 27:22142221.

Ramonell, K., Berrocal-Lobo, M., Koh, S., Wan, J., Edwards, H., Stacey, G., and Somerville, S. 2005. Loss-of-function mutations in chitin responsive genes show increased susceptibility to the powdery mildew pathogen Erysiphe cichoracearum. Plant Physiol. 138:1027-1036.

Robatzek, S., and Somssich, I. E. 2001. A new member of the Arabidopsis WRKY transcription factor family, AtWRKY6, is associated with both senescence- and defence-related processes. Plant J. 28:123-133.

Robatzek, S., and Somssich, I. E. 2002. Targets of AtWRKY6 regulation during plant senescence and pathogen defense. Genes Dev. 16:1139-1149.

Rodriguez, M. C., Petersen, M., and Mundy, J. 2010. Mitogen-activated protein kinase signaling in plants. Annu. Rev. Plant Biol. 61:621-649.

Rosso, M. G., Li, Y., Strizhov, N., Reiss, B., Dekker, K., and Weisshaar, B. 2003. An Arabidopsis thaliana T-DNA mutagenized population (GABIKat) for flanking sequence tag-based reverse genetics. Plant Mol. Biol. 53:247-259.

Shibuya, N., and Minami, E. 2001. Oligosaccharide signalling for defence responses in plant. Physiol. Mol. Plant Pathol. 59:223-233.

Spoel, S. H., Koornneef, A., Claessens, S. M., Korzelius, J. P., Van Pelt, J. A., Mueller, M. J., Buchala, A. J., Metraux, J. P., Brown, R., Kazan, K., Van Loon, L. C., Dong, X., and Pieterse, C. M. 2003. NPR1 modulates cross-talk between salicylate- and jasmonate-dependent defense pathways through a novel function in the cytosol. Plant Cell 15:760-770.

Thomma, B. P., Eggermont, K., Penninckx, I. A., Mauch-Mani, B., Vogelsang, R., Cammue, B. P., and Broekaert, W. F. 1998. Separate jasmonate-dependent and salicylate-dependent defense-response pathways in Arabidopsis are essential for resistance to distinct microbial pathogens. Proc. Natl. Acad. Sci. U.S.A. 95:15107-15111

To, A., Valon, C., Savino, G., Guilleminot, J., Devic, M., Giraudat, J., and Parcy, F. 2006. A network of local and redundant gene regulation governs Arabidopsis seed maturation. Plant Cell 18:1642-1651.

Uhrig, J. F. 2006. Protein interaction networks in plants. Planta 224:771781 .

van Loon, L. C., Geraats, B. P., and Linthorst, H. J. 2006. Ethylene as a modulator of disease resistance in plants. Trends Plant Sci. 11:184-191.

Veronese, P., Nakagami, H., Bluhm, B., Abuqamar, S., Chen, X., Salmeron, J., Dietrich, R. A., Hirt, H., and Mengiste, T. 2006. The membraneanchored BOTRYTIS-INDUCED KINASE1 plays distinct roles in Arabidopsis resistance to nectrotrophic and biotrophic pathogens. Plant Cell 18:257-273.

Wan, J., Zhang, S., and Stacey, G. 2004. Activation of a mitogen-activated protein kinase pathway in Arabidopsis by chitin. Mol. Plant Pathol. 5:125-135.

Wan, J., Zhang, X. C., Neece, D., Ramonell, K. M., Clough, S., Kim, S. Y., Stacey, M. G., and Stacey, G. 2008a. A LysM receptor-like kinase plays a critical role in chitin signaling and fungal resistance in Arabidopsis. Plant Cell 20:471-481.

Wan, J., Zhang, X. C., and Stacey, G. 2008b. Chitin signaling and plant disease resistance. Plant Signal. Behav. 3:831-833.

Wan, J., Son, G. H., Zhang, X., Le, M. H., Hong, J. C., and Stacey, G. 2010. Chitin-mediated signal transduction and plant innate immunity. Chapter 78 in: Biology of Plant-Microbe Interactions. T. A. Hani Antoun, L. Brisson, D. Prevost, and M. Trepanier, eds. International Society for Molecular Plant-Microbe Interactions, St. Paul, MN, U.S.A.

Wang, D., Amornsiripanitch, N., and Dong, X. 2006. A genomic approach to identify regulatory nodes in the transcriptional network of systemic acquired resistance in plants. PLoS Pathog. 2:e123.

Xu, X., Chen, C., Fan, B., and Chen, Z. 2006. Physical and functional interactions between pathogen-induced Arabidopsis WRKY18, WRKY40, and WRKY60 transcription factors. Plant Cell 18:1310-1326.

Zhang, B., Ramonell, K., Somerville, S., and Stacey, G. 2002. Characterization of early, chitin-induced gene expression in Arabidopsis. Mol Plant-Microbe Interact. 15:963-970.

Zhang, S., and Klessig, D.F. 2001. MAPK cascades in plant defense signaling. Trends Plant Sci. 6:520-527.

Zheng, Z., Qamar, S. A., Chen, Z., and Mengiste, T. 2006. Arabidopsis WRKY33 transcription factor is required for resistance to necrotrophic fungal pathogens. Plant J. 48:592-605.

Zhu, J., Zhang, B., Smith, E. N., Drees, B., Brem, R. B., Kruglyak, L., Bumgarner, R. E., and Schadt, E. E. 2008. Integrating large-scale functional genomic data to dissect the complexity of yeast regulatory networks. Nat. Genet. 40:854-861.

Zipfel, C., Kunze, G., Chinchilla, D., Caniard, A., Jones, J. D., Boller, T., and Felix, G. 2006. Perception of the bacterial PAMP EF-Tu by the receptor EFR restricts Agrobacterium-mediated transformation. Cell 125:749-760.

\section{AUTHOR-RECOMMENDED INTERNET RESOURCES}

Human Genome Center's ATTED II database: atted.jp

The Arabidopsis Biological Resource Center homepage: abrc.osu.edu 